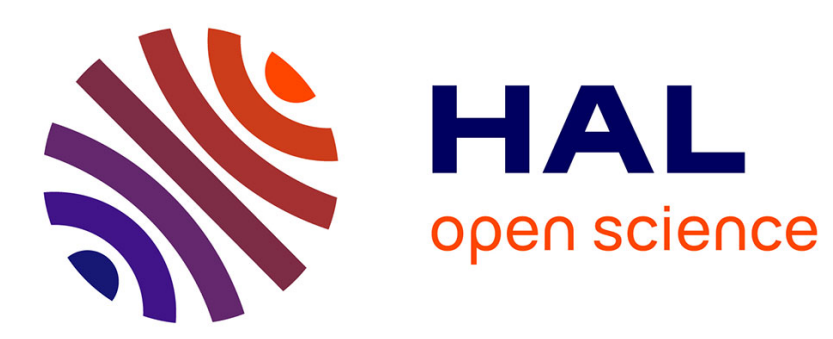

\title{
Capra-Convexity, Convex Factorization and Variational Formulations for the 10 Pseudonorm
}

Jean-Philippe Chancelier, Michel de Lara

\section{To cite this version:}

Jean-Philippe Chancelier, Michel de Lara. Capra-Convexity, Convex Factorization and Variational Formulations for the 10 Pseudonorm. 2021. hal-02459688v2

\section{HAL Id: hal-02459688 \\ https://hal.science/hal-02459688v2}

Preprint submitted on 11 Jan 2021 (v2), last revised 25 Jul 2022 (v4)

HAL is a multi-disciplinary open access archive for the deposit and dissemination of scientific research documents, whether they are published or not. The documents may come from teaching and research institutions in France or abroad, or from public or private research centers.
L'archive ouverte pluridisciplinaire HAL, est destinée au dépôt et à la diffusion de documents scientifiques de niveau recherche, publiés ou non, émanant des établissements d'enseignement et de recherche français ou étrangers, des laboratoires publics ou privés. 


\title{
Capra-Convexity, Convex Factorization and Variational Formulations for the $l_{0}$ Pseudonorm
}

\author{
Jean-Philippe Chancelier and Michel De Lara* \\ CERMICS, Ecole des Ponts, Marne-la-Vallée, France
}

January 11, 2021

\begin{abstract}
The so-called $\ell_{0}$ pseudonorm on the Euclidean space $\mathbb{R}^{d}$ counts the number of nonzero components of a vector. In this paper, we analyze the $l_{0}$ pseudonorm by means of so-called CAPRA conjugacies, for which the underlying source norm and its dual norm are both orthant-strictly monotonic (a notion that we formally introduce and that encompasses the $\ell_{p}$ norms, but for the extreme ones). We obtain three main results. First, we show that the $\ell_{0}$ pseudonorm is equal to its CAPRA-biconjugate, that is, is a CAPRA-convex function. Second, we deduce an unexpected consequence, that we call convex factorization: the $\ell_{0}$ pseudonorm coincides, on the unit sphere of the source norm, with a proper convex lower semicontinuous function. Third, we establish variational formulations for the $\ell_{0}$ pseudonorm by means of generalized top- $k$ dual norms and $k$-support dual norms (that we formally introduce).
\end{abstract}

Key words: $\ell_{0}$ pseudonorm, orthant-strictly monotonic norm, Fenchel-Moreau conjugacy, generalized $k$-support dual norm, sparse optimization.

AMS classification: 46N10, 49N15, 46B99, 52A41, 90C46

\section{Introduction}

The counting function, also called cardinality function or $\ell_{0}$ pseudonorm, counts the number of nonzero components of a vector in $\mathbb{R}^{d}$. It is used in sparse optimization, either as objective function or in the constraints, to obtain solutions with few nonzero entries. However, the mathematical expression of the $\ell_{0}$ pseudonorm makes it difficult to be handled as such in optimization problems. This is why most of the literature on sparse optimization resorts to substitute problems, obtained either from estimates (inequalities) for the $\ell_{0}$ pseudonorm, or from alternative sparsity-inducing terms (especially suitable norms). The literature on sparsity-inducing norms is huge, and we just point out a very succint part of it. We refer

\footnotetext{
*michel.delara@enpc.fr
} 
the reader to [16] that provides a brief tour of the literature dealing with least squares minimization constrained by $k$-sparsity, and to [9] for a survey of the rank function of a matrix, that shares many properties with the $\ell_{0}$ pseudonorm. We refer the reader to [2] for the support norm, to [23] (and references therein) for top norms, and to [13] for generalizations.

Our approach to tackle the $\ell_{0}$ pseudonorm uses so-called CAPRA conjugacies, introduced in [4]. More precisely, in [4], we brought out the class of couplings CAPRA (dependending on an underlying source norm) and we established expressions for CAPRA-conjugates and biconjugates, and CAPRA-subdifferentials of nondecreasing functions of the $\ell_{0}$ pseudonorm. In [5], we introduced the coupling E-CAPRA related to the Euclidean norm and we showed that the $\ell_{0}$ pseudonorm is E-CAPRA-convex and displays hidden convexity in the following sense. The $\ell_{0}$ pseudonorm satisfies a convex factorization property: it can be written as the composition of a convex lsc function (on the Euclidean space) with the normalization mapping that maps any nonzero vector onto the Euclidean unit sphere, hence it coincides with a convex lsc function on the Euclidean unit sphere.

In this paper, we go beyond the two above papers in several directions. We generalize the results of [5] by showing that not only the $\ell_{0}$ pseudonorm but any nondecreasing function of the $\ell_{0}$ pseudonorm is CAPRA-convex and displays hidden convexity (convex factorization property), and not only for the Euclidean norm but for a class of norms that encompasses it (including the $\ell_{p}$-norms for $\left.p \in\right] 1, \infty[$ ). Moreover, we extend the hidden convexity property to subdifferentials. Indeed, we show that the CAPRA-subdifferential of a nondecreasing function of the $\ell_{0}$ pseudonorm coincides, on the unit sphere, with the Rockafellar-Moreau subdifferential of the associated convex lsc function (in the convex factorization property). Whereas we obtained CAPRA-convex lower bounds (inequalities) in [4], for nondecreasing functions of the $\ell_{0}$ pseudonorm, we now obtain identities. Whereas we obtained an expression for the CAPRA-subdifferential of a nondecreasing function of the $\ell_{0}$ pseudonorm, we now prove that it is not empty.

The paper is organized as follows. In Sect. 2, we provide background on the $\ell_{0}$ pseudonorm and CAPRA-conjugacies. We also introduce a new class of orthant-strictly monotonic norms, as well as sequences of generalized top- $k$ and $k$-support dual norms. We show that nondecreasing functions of the $\ell_{0}$ pseudonorm are CAPRA-convex. In Sect. 3, we show that any nondecreasing function of the $\ell_{0}$ pseudonorm coincides, when restricted to the unit sphere, with a proper convex lsc function. Then, we deduce variational formulations for nondecreasing functions of the $\ell_{0}$ pseudonorm which involve the sequence of generalized $k$-support dual norms. Appendix A gathers background on properties of norms that are relevant for the $\ell_{0}$ pseudonorm, and Appendix B gathers background on the Fenchel conjugacy.

\section{Capra-convexity of the $\ell_{0}$ pseudonorm with orthant- strictly monotonic norms}

In $\S 2.1$, we provide background on the $\ell_{0}$ pseudonorm and on the family of CAPRA conjugacies (introduced in [4]). Then, in $\S 2.2$, we introduce norms that are especially relevant for the 
$\ell_{0}$ pseudonorm, like orthant-strictly monotonic norms. Finally, in $\S 2.3$, we prove that the $\ell_{0}$ pseudonorm is CAPRA-convex when the underlying norm and its dual norm are both orthant-strictly monotonic.

\subsection{Background on the $\ell_{0}$ pseudonorm and the Capra conjugacy}

We work on the Euclidean space $\mathbb{R}^{d}$ (where $d$ is a nonzero integer), equipped with the scalar product $\langle\cdot, \cdot\rangle$ (but not necessarily with the Euclidean norm). We use the notation $\llbracket j, k \rrbracket=\{j, j+1, \ldots, k-1, k\}$ for any pair of integers such that $j \leq k$.

Let $\|\cdot \mid\|$ be a norm on $\mathbb{R}^{d}$, that we will call the source norm. We denote the unit sphere $\mathbb{S}$ and the unit ball $\mathbb{B}$ of the source norm $\|\cdot\| \mid \|$ by

$$
\mathbb{S}=\left\{x \in \mathbb{R}^{d} \mid\|x\|=1\right\}, \mathbb{B}=\left\{x \in \mathbb{R}^{d} \mid\|x\| \leq 1\right\}
$$

For any vector $x \in \mathbb{R}^{d}, \operatorname{supp}(x)=\left\{j \in \llbracket 1, d \rrbracket \mid x_{j} \neq 0\right\} \subset \llbracket 1, d \rrbracket$ is the support of $x$. The so-called $\ell_{0}$ pseudonorm is the function $\ell_{0}: \mathbb{R}^{d} \rightarrow \llbracket 0, d \rrbracket$ defined by

$$
\ell_{0}(x)=|\operatorname{supp}(x)|=\text { number of nonzero components of } x, \forall x \in \mathbb{R}^{d},
$$

where $|K|$ denotes the cardinality of a subset $K \subset \llbracket 1, d \rrbracket$. The $\ell_{0}$ pseudonorm shares three out of the four axioms of a norm: nonnegativity, positivity except for $x=0$, subadditivity. The axiom of 1-homogeneity does not hold true. By contrast, the $\ell_{0}$ pseudonorm is 0 homogeneous:

$$
\ell_{0}(\rho x)=\ell_{0}(x), \forall \rho \in \mathbb{R} \backslash\{0\}, \forall x \in \mathbb{R}^{d} .
$$

Following [4], we introduce the coupling CAPRA.

Definition 1 ([4, Definition 8]) The constant along primal rays coupling $c: \mathbb{R}^{d} \times \mathbb{R}^{d} \rightarrow \mathbb{R}$, or CAPRA, between $\mathbb{R}^{d}$ and itself, is the function

$$
c:(x, y) \in \mathbb{R}^{d} \times \mathbb{R}^{d} \mapsto\left\{\begin{array}{lc}
\frac{\langle x, y\rangle}{\|x\|}, & x \neq 0 \\
0, & \text { else. }
\end{array}\right.
$$

We stress the point that, in (4), the Euclidean scalar product $\langle x, y\rangle$ and the norm term $\|x\|$ need not be related, that is, the norm $\|\cdot\| \cdot \|$ is not necessarily the Euclidean norm.

The coupling CAPRA has the property of being constant along primal rays, hence the acronym CAPRA (Constant Along Primal RAys). We introduce the primal normalization mapping $n: \mathbb{R}^{d} \rightarrow \mathbb{S} \cup\{0\}$, from $\mathbb{R}^{d}$ towards the unit sphere $\mathbb{S}$ united with $\{0\}$, as follows:

$$
n: x \in \mathbb{R}^{d} \mapsto \begin{cases}\frac{x}{\|x\|} & x \neq 0 \\ 0, & \text { else. }\end{cases}
$$

Now, we introduce notions and notation from generalized convexity [22, 21, 12]. As we manipulate functions with values in $\overline{\mathbb{R}}=[-\infty,+\infty]$, we adopt the Moreau lower and upper 
additions [15] that extend the usual addition with $(+\infty)+(-\infty)=(-\infty)+(+\infty)=-\infty$ or with $(+\infty) \dot{+}(-\infty)=(-\infty) \dot{+}(+\infty)=+\infty$. The $\dot{c}$-Fenchel-Moreau conjugate of a function $f: \mathbb{R}^{d} \rightarrow \overline{\mathbb{R}}$, with respect to the coupling $\dot{c}$, is the function $f^{\dot{c}}: \mathbb{R}^{d} \rightarrow \overline{\mathbb{R}}$ defined by

$$
f^{\dot{\varphi}}(y)=\sup _{x \in \mathbb{R}^{d}}(\dot{\zeta}(x, y)+(-f(x))), \forall y \in \mathbb{R}^{d} .
$$


is the function $f^{c c^{\prime}}: \mathbb{R}^{d} \rightarrow \overline{\mathbb{R}}$ defined by

$$
f^{\dot{c} \dot{c}^{\prime}}(x)=\sup _{y \in \mathbb{R}^{d}}\left(\dot{\phi}(x, y)+\left(-f^{\dot{\phi}}(y)\right)\right), \forall x \in \mathbb{R}^{d} .
$$

The biconjugate of a function $f: \mathbb{R}^{d} \rightarrow \overline{\mathbb{R}}$ satisfies the inequality

$$
f^{\mathrm{c} \dot{\varphi}^{\prime}}(x) \leq f(x), \forall x \in \mathbb{R}^{d} .
$$

When the coupling $\boldsymbol{c}$ is replaced by the Euclidean scalar product $\langle\cdot, \cdot\rangle$, we recover well-known expressions of the Fenchel conjugacy (see Appendix B).

\subsection{Relevant norms for the $\ell_{0}$ pseudonorm}

In $\S 2.2 .1$, we recall the notions of restriction norms and of generalized coordinate- $k$ and dual coordinate- $k$ norms. In $\S 2.2 .2$, we introduce two new families of norms, the generalized top- $k$ and $k$-support dual norms. Finally, in $\S 2.2 .3$, we define a new class of orthant-strictly monotonic norms.

\subsubsection{Restriction norms and generalized coordinate- $k$ and dual coordinate- $k$ norms}

For any subset $K \subset \llbracket 1, d \rrbracket$, we define the subspace

$$
\mathcal{R}_{K}=\left\{x \in \mathbb{R}^{d} \mid x_{j}=0, \forall j \notin K\right\} \subset \mathbb{R}^{d}
$$

with $\mathcal{R}_{\emptyset}=\{0\}$, and then three norms on the subspace $\mathcal{R}_{K}$ of $\mathbb{R}^{d}$, as follows.

- The $K$-restriction norm $\|\cdot\|_{K}$ is defined by $\|x\|_{K}=\|x\|$, for any $x \in \mathcal{R}_{K}$.

- The $(\star, K)$-norm $\|\cdot\|_{\star, K}$ is the norm $\left(\|\cdot \mid\|_{\star}\right)_{K}$, given by the restriction to the subspace $\mathcal{R}_{K}$ of the dual norm $\|\cdot\|_{\star}$ (first dual, as recalled in definition (26) of a dual norm, then restriction),

- The $(K, \star)$-norm $\|\cdot \cdot\|_{K, \star}$ is the norm $\left(\|\cdot\|_{K}\right)_{\star}$, given by the dual norm (on the subspace $\mathcal{R}_{K}$ ) of the restriction norm $\|\cdot \cdot\|_{K}$ to the subspace $\mathcal{R}_{K}$ (first restriction, then dual). 
For any $x \in \mathbb{R}^{d}$ and subset $K \subset \llbracket 1, d \rrbracket$, we denote by $x_{K} \in \mathcal{R}_{K} \subset \mathbb{R}^{d}$ the vector which coincides with $x$, except for the components outside of $K$ that vanish (this definition is valid for $K=\emptyset$, giving $\left.x_{\emptyset}=0 \in \mathcal{R}_{\emptyset}=\{0\}\right)$.

Definition 2 ([4, Definition 3]) For $k \in \llbracket 1, d \rrbracket$, we call generalized coordinate- $k$ norm the norm $\|\cdot\|_{(k)}^{\mathcal{R}}$ on $\mathbb{R}^{d}$ whose dual norm is the generalized dual coordinate- $k$ norm, denoted by $\|\cdot\|_{(k), \star}^{\mathcal{R}}$, with expression ${ }^{1}$

$$
\|y\|_{(k), \star}^{\mathcal{R}}=\sup _{|K| \leq k}\left\|y_{K}\right\|_{K, \star}, \quad \forall y \in \mathbb{R}^{d} .
$$

We denote the unit sphere $\mathbb{S}_{(k), \star}^{\mathcal{R}}$ and the unit ball $\mathbb{B}_{(k), \star}^{\mathcal{R}}$ by

$$
\mathbb{S}_{(k), \star}^{\mathcal{R}}=\left\{y \in \mathbb{R}^{d} \mid\|y\|_{(k), \star}^{\mathcal{R}}=1\right\}, \mathbb{B}_{(k), \star}^{\mathcal{R}}=\left\{y \in \mathbb{R}^{d} \mid\|y\|_{(k), \star}^{\mathcal{R}} \leq 1\right\}, \quad \forall k \in \llbracket 1, d \rrbracket .
$$

We give examples of generalized coordinate- $k$ and dual coordinate- $k$ norms in [4, Table 1].

\subsubsection{Generalized top- $k$ and $k$-support dual norms}

We introduce two new families of norms, that we call generalized top- $k$ and $k$-support dual norms.

Definition 3 For $k \in \llbracket 1, d \rrbracket$, we call generalized top- $k$ dual norm (associated with the source norm $\|\cdot\| \mid \|)$ the norm on $\mathbb{R}^{d}$ defined by ${ }^{2}$

$$
\|y\|_{\star,(k)}^{\mathrm{tn}}=\sup _{|K| \leq k}\left\|y_{K}\right\|_{\star}=\sup _{|K| \leq k}\left\|y_{K}\right\|_{\star, K}, \quad \forall y \in \mathbb{R}^{d} .
$$

We call generalized $k$-support dual norm the dual norm (on $\mathbb{R}^{d}$ ) of the generalized top- $k$ dual norm, denoted by ${ }^{3}$

$$
\|\cdot\|_{\star,(k)}^{\star \mathrm{sn}}=\left(\|\| \cdot \|_{\star,(k)}^{\mathrm{tn}}\right)_{\star}, \quad \forall k \in \llbracket 1, d \rrbracket,
$$

with unit sphere $\mathbb{S}_{\star,(k)}^{\star s n}$ and unit ball $\mathbb{B}_{\star,(k)}^{\star s n}$ given by

$$
\mathbb{S}_{\star,(k)}^{\star \mathrm{n}}=\left\{x \in \mathbb{R}^{d} \mid\|x\|_{*,(k)}^{\star \mathrm{sn}}=1\right\}, \quad \mathbb{B}_{\star,(k)}^{\star \mathrm{sn}}=\left\{x \in \mathbb{R}^{d} \mid\|x\|_{*,(k)}^{\star \mathrm{sn}} \leq 1\right\}, \quad \forall k \in \llbracket 1, d \rrbracket .
$$

We now give examples of generalized top- $k$ and $k$-support dual norms in the case of $\ell_{p}$ source norm. We recall that the $\ell_{p}$-norms $\|\cdot\|_{p}$ on the space $\mathbb{R}^{d}$ are defined by $\|x\|_{p}=$ $\left(\sum_{i=1}^{d}\left|x_{i}\right|^{p}\right)^{\frac{1}{p}}$ for $p \in\left[1, \infty\left[\right.\right.$, and by $\|x\|_{\infty}=\sup _{i \in \llbracket 1, d \rrbracket}\left|x_{i}\right|$. It is well-known that the dual

\footnotetext{
${ }^{1}$ The notation $\sup _{|K| \leq k}$ is a shorthand for $\sup _{K \subset \llbracket 1, d \rrbracket,|K| \leq k}$.

${ }^{2}$ We adopt the convention $\|\cdot\|_{\star,(0)}^{\mathrm{tn}}=0$ (although this is not a norm, but a seminorm).

${ }^{3}$ We adopt the convention $\|\cdot\|_{*,(0)}^{* \mathrm{sn}}=0$ (although this is not a norm, but a seminorm). We use the symbol $\star$ in the superscript to indicate that the generalized $k$-support dual norm $\|\cdot\|_{\star,(k)}^{\star \star s n}$ is a dual norm. To stress the point, we use the letter $x$ for a primal vector, like in $\|x\|_{\star,(k)}^{\star \mathrm{sn}}$, and the letter $y$ for a dual vector, like in $\|y\|_{\star,(k)}^{\mathrm{tn}}$.
} 
norm of the norm $\|\cdot\|_{p}$ is the $\ell_{q}$-norm $\|\cdot\|_{q}$, where $q$ is such that $1 / p+1 / q=1$ (with the extreme cases $q=\infty$ when $p=1$, and $q=1$ when $p=\infty$ ).

We start with a Lemma, whose proof is easy. For any $y \in \mathbb{R}^{d}$, we denote by $|y|=$ $\left(\left|y_{1}\right|, \ldots,\left|y_{d}\right|\right)$ the vector of $\mathbb{R}^{d}$ with components $\left|y_{i}\right|, i \in \llbracket 1, d \rrbracket$. Letting $y \in \mathbb{R}^{d}$ and $\nu$ be a permutation of $\llbracket 1, d \rrbracket$ such that $\left|y_{\nu(1)}\right| \geq\left|y_{\nu(2)}\right| \geq \cdots \geq\left|y_{\nu(d)}\right|$, we note $y^{\downarrow}=$ $\left(\left|y_{\nu(1)}\right|,\left|y_{\nu(2)}\right|, \ldots,\left|y_{\nu(d)}\right|\right)$.

Lemma 4 Let $\|\cdot\| \cdot \|$ be a norm on $\mathbb{R}^{d}$. Then, if the norm $\|\cdot\|$ is permutation invariant and monotonic (with respect to the partial order on $\mathbb{R}^{d}$ given by the nonnegative orthant cone), we have that $\|y\|_{\star,(k)}^{\text {tn }}=\left\|y_{\{1, \ldots, k\}}^{\downarrow}\right\|_{\star}$, where $y_{\{1, \ldots, k\}}^{\downarrow} \in \mathbb{R}^{d}$ is given by $\left(y^{\downarrow}\right)_{\{1, \ldots, k\}}$, for all $y \in \mathbb{R}^{d}$.

We start with generalized top- $k$ dual norms as in (10) (see the second column of Table 1). When the norm $\|\cdot\| \cdot \|$ is the Euclidean norm $\|\cdot\|_{2}$ of $\mathbb{R}^{d}$, the generalized top- $k$ dual norm is known under different names: the top- $(k, 2)$ norm in $[23$, p. 8$]$, or the 2 - $k$-symmetric gauge norm [14] or the Ky Fan vector norm [17]. Indeed, in all these cases, the norm of a vector $y$ is obtained with a subvector of size $k$ having the $k$ largest components in module, because the assumptions of Lemma 4 are satisfied. More generally, when the norm $\|\cdot\| \|$ is the $\ell_{p}$-norm $\|\cdot\|_{p}$, for $p \in[1, \infty]$, the assumptions of Lemma 4 are also satisfied, as $\ell_{p}$-norms are permutation invariant and monotonic. Therefore, we obtain that the corresponding generalized top- $k$ dual norm $\left(\|\cdot\|_{p}\right)_{\star,(k)}^{\text {tn }}$ has the expression $\left(\|\cdot\|_{p}\right)_{\star,(k)}^{\text {tn }}(y)=\sup _{|K| \leq k}\left\|y_{K}\right\|_{q}=\left\|y_{\{1, \ldots, k\}}^{\downarrow}\right\|_{q}$, for all $y \in \mathbb{R}^{d}$, and where $1 / p+1 / q=1$. We call ${ }^{4}$ top- $(q, k)$ norm — denoted by $\|\cdot\|_{q, k}^{\text {tn }}$ - the generalized top- $k$ dual norm $\|\cdot\|_{\star,(k)}^{\mathrm{tn}}$ in (10) when the source norm $\|\cdot\| \cdot \|$ is the $\ell_{p}$-norm $\|\cdot\|_{p}$. Notice that $\|\cdot\|_{\infty, k}^{\text {tn }}=\|\cdot\|_{\infty}$ for all $k \in \llbracket 1, d \rrbracket$.

Now, we turn to generalized $k$-support dual norms as in (11) (see the third column of Table 1). When the norm $\|\cdot\| \cdot \|$ is the Euclidean norm $\|\cdot\|_{2}$ of $\mathbb{R}^{d}$, the generalized $k$ support norm is the so-called $k$-support norm [2]. More generally, in [13, Definition 21], the authors define the $k$-support $p$-norm or $(p, k)$-support norm for $p \in[1, \infty]$. They show, in $\left[13\right.$, Corollary 22], that the dual norm $\left(\left(\|\cdot\|_{p}\right)_{(k)}^{\mathrm{tn}}\right)_{\star}$ of the above top- $(p, k)$ norm is the $(q, k)$-support norm, where $1 / p+1 / q=1$. Therefore, the generalized $k$-support dual norm in (11) is the $(p, k)$-support norm — denoted by $\|\cdot\|_{p, k}^{\mathrm{sn}}$ — when the source norm $\|\cdot\| \cdot \|$ is the $\ell_{p}$-norm $\|\cdot\|_{p}$, for $p \in[1, \infty]$. The formula $\|x\|_{\infty, k}^{\text {sn }}=\max \left\{\|x\|_{1} / k,\|x\|_{\infty}\right\}$ can be found in $[3$, Exercise IV.1.18, p. 90].

\subsubsection{Orthant-monotonic and orthant-strictly monotonic norms}

We recall the definition of orthant-monotonic norms and we introduce the new one of orthantstrictly monotonic norms, that will prove especially relevant for the $\ell_{0}$ pseudonorm.

Definition 5 A norm $\|\cdot \mid\|$ on the space $\mathbb{R}^{d}$ is called

\footnotetext{
${ }^{4}$ We invert the indices in the naming convention of $[23$, p. 5, p. 8$]$, where top- $(k, 1)$ and top- $(k, 2)$ were used.
} 


\begin{tabular}{|c|c|c|}
\hline source norm $\|\cdot\| \|$ & \|\|$\cdot \|_{\star,(k)}^{\operatorname{tn}}, k \in \llbracket 1, d \rrbracket$ & \|\|$\cdot \|_{\star,(k)}^{\star \mathrm{sn}}, k \in \llbracket 1, d \rrbracket$ \\
\hline$\|\cdot\|_{p}$ & $\begin{array}{c}\text { top- } \\
(q, k) \text { norm } \\
\|y\|_{q, k}^{\text {tn }} \\
\|y\|_{q, k}^{\text {tn }}=\left(\sum_{l=1}^{k}\left|y_{\nu(l)}\right|^{q}\right)^{\frac{1}{q}}\end{array}$ & $\begin{array}{c}(p, k) \text {-support norm } \\
\qquad\|x\|_{p, k}^{\mid \mathrm{s} n} \\
\text { no analytic expression }\end{array}$ \\
\hline$\|\cdot\|_{1}$ & $\begin{array}{c}\text { top- }(\infty, k) \text { norm } \\
\ell_{\infty} \text {-norm } \\
\|y\|_{\infty, k}^{\text {tn }}=\|y\|_{\infty}, \forall k \in \llbracket 1, d \rrbracket\end{array}$ & $\begin{array}{c}(1, k) \text {-support norm } \\
\ell_{1} \text {-norm } \\
\|x\|_{1, k}^{\mathrm{sn}}=\|x\|_{1}, \forall k \in \llbracket 1, d \rrbracket\end{array}$ \\
\hline$\|\cdot\|_{2}$ & $\begin{array}{c}\text { top- }(2, k) \text { norm } \\
\|y\|_{2, k}^{\mathrm{tn}}=\sqrt{\sum_{l=1}^{k}\left|y_{\nu(l)}\right|^{2}} \\
\|y\|_{2,1}^{\mathrm{tn}}=\|y\|_{\infty}\end{array}$ & $\begin{array}{c}(2, k) \text {-support norm } \\
\|x\|_{2, k}^{\mathrm{sn}} \text { no analytic expression } \\
(\text { computation }[2, \text { Prop. 2.1]) } \\
\|x\|_{2,1}^{\mathrm{sn}}=\|x\|_{1}\end{array}$ \\
\hline$\|\cdot\|_{\infty}$ & $\begin{array}{c}\text { top- }(1, k) \text { norm } \\
\|y\|_{1, k}^{\text {tn }}=\sum_{l=1}^{k}\left|y_{\nu(l)}\right| \\
\|y\|_{1,1}^{\text {tn }}=\|y\|_{\infty}\end{array}$ & $\begin{array}{c}(\infty, k) \text {-support norm } \\
\|x\|_{\infty, k}^{\text {sn }}=\max \left\{\frac{\|x\|_{1}}{k},\|x\|_{\infty}\right\} \\
\|x\|_{1,1}^{\text {sn }}=\|x\|_{1}\end{array}$ \\
\hline
\end{tabular}

Table 1: Examples of generalized top- $k$ and $k$-support dual norms generated by the $\ell_{p}$ source norms $\|\cdot\|\|=\| \cdot \|_{p}$ for $p \in[1, \infty]$, where $1 / p+1 / q=1$. For $y \in \mathbb{R}^{d}, \nu$ denotes a permutation of $\{1, \ldots, d\}$ such that $\left|y_{\nu(1)}\right| \geq\left|y_{\nu(2)}\right| \geq \cdots \geq\left|y_{\nu(d)}\right|$. 
- orthant-monotonic [8, Definition 2.6] if, for all $x, x^{\prime}$ in $\mathbb{R}^{d}$, we have $\left(|x| \leq\left|x^{\prime}\right|\right.$ and $x \circ x^{\prime} \geq$ $0 \Rightarrow\left\|\left|\||\leq|\| x^{\prime} \|\right|\right)$, where $|x| \leq\left|x^{\prime}\right|$ means $\left|x_{i}\right| \leq\left|x_{i}^{\prime}\right|$ for all $i \in \llbracket 1, d \rrbracket$, and where $x \circ x^{\prime}=\left(x_{1} x_{1}^{\prime}, \ldots, x_{d} x_{d}^{\prime}\right)$ is the Hadamard (entrywise) product,

- orthant-strictly monotonic if, for all $x, x^{\prime}$ in $\mathbb{R}^{d}$, we have $\left(|x|<\left|x^{\prime}\right|\right.$ and $x \circ x^{\prime} \geq$ $\left.0 \Rightarrow\|x\|\left|<\left\|x^{\prime}\right\|\right|\right)$, where $|x|<\left|x^{\prime}\right|$ means that $\left|x_{i}\right| \leq\left|x_{i}^{\prime}\right|$ for all $i \in \llbracket 1, d \rrbracket$, and there exists $j \in \llbracket 1, d \rrbracket$, such that $\left|x_{j}\right|<\left|x_{j}^{\prime}\right|$.

All the $\ell_{p}$-norms $\|\cdot\|_{p}$ on the space $\mathbb{R}^{d}$, for $p \in[1, \infty[$, are strictly monotonic, hence orthantstrictly monotonic. By contrast, the $\ell_{\infty}$-norm $\|\cdot\|_{\infty}$ is orthant-monotonic but not orthantstrictly monotonic.

\subsection{Capra-convexity of the $\ell_{0}$ pseudonorm}

The main result of this Section is Theorem 6 which states that, when both the source norm $\|\cdot\| \cdot \|$ and its dual norm $\|\cdot\| \cdot \|_{\star}$ are orthant-strictly monotonic, then any nondecreasing function of the $\ell_{0}$ pseudonorm is equal to its CAPRA-biconjugate, that is, is a CAPRA-convex function. This considerably generalizes the result in [5, Theorem 3.5], which was established for the Euclidean norm and only for the $\ell_{0}$ pseudonorm. The proof of Theorem 6 relies on Proposition 7, stated and proved after, which establishes the nonemptiness of a suitable CAPRA-subdifferential. In [4, Equation (32)], we define the CAPRA-subdifferential of the function $f: \mathbb{R}^{d} \rightarrow \overline{\mathbb{R}}$ at $x \in \mathbb{R}^{d}$ by

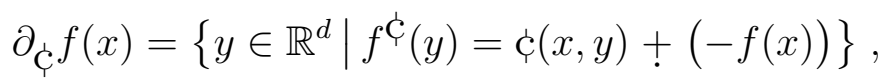



Theorem 6 Let $\|\cdot \mid\|$ be a norm on $\mathbb{R}^{d}$ with associated sequence $\left\{\|\mid \cdot\|_{\star,(j)}^{\text {tn }}\right\}_{j \in \llbracket 1, d \rrbracket}$ of generalized top-k dual norms, as in Definition 3, and with associated CAPRA coupling $c$ as in (4). If the norm $\mid\|\cdot\|$ is orthant-monotonic, then, for any function $\varphi: \llbracket 0, d \rrbracket \rightarrow \mathbb{R}$, we have ${ }^{5}$

$$
\left(\varphi \circ \ell_{0}\right)^{c}=\sup _{j \in \llbracket 0, d \rrbracket}\left[\|\cdot\|_{\star,(j)}^{\text {tn }}-\varphi(j)\right] .
$$

If both the norm $\|\cdot\| \cdot \|$ and the dual norm $\|\cdot \cdot\|_{\star}$ are orthant-strictly monotonic, then, for any nondecreasing function $\varphi: \llbracket 0, d \rrbracket \rightarrow \mathbb{R}$, we have

$$
\left(\varphi \circ \ell_{0}\right)^{\phi c^{\prime}}=\varphi \circ \ell_{0}
$$

\footnotetext{
${ }^{5}$ We recall the convention that $\|\cdot \cdot\|_{\star,(0)}^{\text {tn }}=0$.
} 
Proof. Suppose that the norm $\|\cdot\| \|$ is orthant-monotonic. Then, by (31) in Proposition 13 (Appendix A), we get that $\|\cdot\|_{(k)}^{\mathcal{R}}=\|\cdot\|_{\star,(k)}^{\star \mathrm{sn}}$ and $\|\cdot\|_{(k), \star}^{\mathcal{R}}=\|\cdot\|_{\star,(k)}^{\mathrm{tn}}$. Moreover, it is proved in [4, Equation (33) in Proposition 11] that $\left(\varphi \circ \ell_{0}\right)^{\mathrm{C}}=\sup _{j \in \llbracket 0, d \rrbracket}\left[\|\cdot\|_{(j), \star}^{\mathcal{R}}-\varphi(j)\right]$. As $\|\cdot\|_{(j), \star}^{\mathcal{R}}=\|\cdot\|_{\star,(j)}^{\mathrm{tn}}$, we obtain (14).

Suppose that both the norm $\|\cdot \cdot\|$ and the dual norm $\|\cdot \cdot\|_{\star}$ are orthant-strictly monotonic. Then, the forthcoming Proposition 7 applies. Therefore, for any vector $x \in \mathbb{R}^{d}$ and any $y \in \partial_{\dot{C}}\left(\varphi \circ \ell_{0}\right)(x) \neq$ $\emptyset$, we obtain

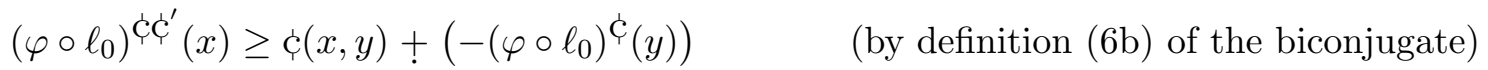

$$
\begin{aligned}
& =\dot{\phi}(x, y)-\left(\varphi \circ \ell_{0}\right)^{\dot{\phi}}(y) \\
& \text { (because }-\infty<c(x, y)<+\infty \text { by }(4) \text {, so that the usual addition applies) } \\
& =\dot{c}(x, y)-\left(\dot{c}(x, y)-\left(\varphi \circ \ell_{0}\right)(x)\right)
\end{aligned}
$$

by definition (13) of the CAPRA-subdifferential $\partial_{\dot{c}}\left(\varphi \circ \ell_{0}\right)(x)$, and where again we can use the usual addition

$$
=\left(\varphi \circ \ell_{0}\right)(x) .
$$

On the other hand, we have that $\left(\varphi \circ \ell_{0}\right)^{\mathrm{c} \boldsymbol{c}^{\prime}}(x) \leq\left(\varphi \circ \ell_{0}\right)(x)$ by $(6 \mathrm{c})$. We conclude that $\left(\varphi \circ \ell_{0}\right)^{\mathrm{c} \boldsymbol{c}^{\prime}}(x)=$ $\left(\varphi \circ \ell_{0}\right)(x)$, which is $(15)$.

This ends the proof.

Now, we prove that the CAPRA-subdifferential of any nondecreasing function of the $\ell_{0}$ pseudonorm is not empty. ${ }^{6}$

Proposition 7 Let $\|\cdot\| \mid \|$ be a norm on $\mathbb{R}^{d}$ with associated CAPRA coupling $c$ as in (4). If both the norm $\|\cdot\| \mid \|$ and the dual norm $\|\cdot\|_{\star}$ are orthant-strictly monotonic, and if $\varphi: \llbracket 0, d \rrbracket \rightarrow \mathbb{R}$ is a nondecreasing function, then

$$
\partial_{\mathcal{C}}\left(\varphi \circ \ell_{0}\right)(x) \neq \emptyset, \quad \forall x \in \mathbb{R}^{d}
$$

More precisely, when $x=0$, we have that $\partial_{\mathcal{C}}\left(\varphi \circ \ell_{0}\right)(0)=\bigcap_{j \in \llbracket 1, d \rrbracket}[\varphi(j)-\varphi(0)] \mathbb{B}_{(j), \star}^{\mathcal{R}} \neq \emptyset$, where the unit ball $\mathbb{B}_{(k), \star}^{\mathcal{R}}$ is defined in (9). When $x \neq 0$, there exists $y \in \mathbb{R}^{d}$ satisfying $\operatorname{supp}(y)=\operatorname{supp}(x)$ and $\langle x, y\rangle=\|x\| \times\|y\|_{\star}$, and for all such $y \in \mathbb{R}^{d}$ we have that $\lambda y \in$ $\partial_{\mathcal{C}}\left(\varphi \circ \ell_{0}\right)(x)$ for $\lambda>0$ large enough.

\footnotetext{
${ }^{6}$ In [9, Section 8], it is shown that all the generalized (Fenchel) subdifferentials (proximal, Fréchet, viscosity, limiting, Clarke) of the rank function coincide and define a vector space. By contrast, the CAPRAsubdifferential of the $\ell_{0}$ pseudonorm is not a vector space, but we can show that it is closed convex. Indeed, it is easily seen that (13) can also be written as $\partial_{\dot{c}} f(x)=\left\{y \in \mathbb{R}^{d} \mid f^{\dot{c}}(y) \leq \grave{c}(x, y)+(-f(x))\right\}$, where the function $f^{\dot{C}}$ is closed convex by [4, Equation (30b) in Proposition 9], hence $\partial_{\dot{C}} f(x)$ is a closed convex set.
} 
Proof. Since the norm $\|\cdot \cdot\|$ is orthant-strictly monotonic, it is orthant-monotonic, so that we have $\|\cdot\|_{(j)}^{\mathcal{R}}=\|\| \cdot \|_{\star,(j)}^{\star \star s n}$ and $\|\cdot\|_{(j), \star}^{\mathcal{R}}=\|\cdot\|_{\star,(j)}^{\mathrm{tn}}$, for $j \in \llbracket 0, d \rrbracket$ by (31) in Proposition 13 (with the convention that these are the null seminorms in the case $j=0$ ). Therefore, we can translate all the results with generalized top- $k$ and $k$-support dual norms (Definition 3 ) instead of coordinate- $k$ and dual coordinate- $k$ norms (Definition 2).

When $x=0$, we have by [4, Equation (39) in Proposition 14] that

$$
\partial_{\dot{C}}\left(\varphi \circ \ell_{0}\right)(0)=\bigcap_{j \in \llbracket 1, d \rrbracket}[\varphi(j) \dot{+}(-\varphi(0))] \mathbb{B}_{(j), \star}^{\mathcal{R}}=\bigcap_{j \in \llbracket 1, d \rrbracket}[\varphi(j)-\varphi(0)] \mathbb{B}_{(j), \star}^{\mathcal{R}} \ni 0,
$$

because $\varphi(j) \dot{+}(-\varphi(0))=\varphi(j)-\varphi(0) \geq 0$ since $\varphi: \llbracket 0, d \rrbracket \rightarrow \mathbb{R}$ is a nondecreasing function.

From now on, we consider $x \in \mathbb{R}^{d} \backslash\{0\}$ such that $\ell_{0}(x)=l \in \llbracket 1, d \rrbracket$, and we will use the following equivalence, established in [4, Equation (40) in Proposition 14]

$$
y \in \partial_{\dot{C}}\left(\varphi \circ \ell_{0}\right)(x) \Longleftrightarrow\left\{\begin{array}{l}
y \in N_{\mathbb{B}_{(l)}^{\mathcal{R}}}\left(\frac{x}{\|x\|_{(l)}^{\mathcal{R}}}\right) \\
\text { and } l \in \arg \max _{j \in \llbracket 0, d \rrbracket}\left[\|y\|_{(j), \star}^{\mathcal{R}}-\varphi(j)\right],
\end{array}\right.
$$

where the normal cone $N_{\mathbb{B}_{(l)}^{\mathcal{R}}}\left(\frac{x}{\|x\|_{(l)}^{\mathcal{R}}}\right)$ is defined in (28).

Since the norm $\|\cdot\| \cdot \|$ is orthant-strictly monotonic, we know by Item $(\boldsymbol{b})$ in Proposition 12 that there exists a (nonzero) vector $y \in \mathbb{R}^{d}$ such that

$$
\begin{aligned}
L=\operatorname{supp}(x)=\operatorname{supp}(y) \text { hence } \ell_{0}(y) & =\ell_{0}(x)=l>1, \\
\langle x, y\rangle & =\|x\| \times\|y\|_{\star} .
\end{aligned}
$$

Since the dual norm $\|\cdot\|_{\star}$ is orthant-strictly monotonic, we know by (32) in Proposition 14 that

$$
\|y\|_{(1), \star}^{\mathcal{R}}<\cdots<\|y\|_{(l-1), \star}^{\mathcal{R}}<\|y\|_{(l), \star}^{\mathcal{R}}=\cdots=\|y\|_{(d), \star}^{\mathcal{R}}=\|y\|_{\star} .
$$

We now show that $y \in \partial_{\dot{c}}\left(\varphi \circ \ell_{0}\right)(x)$.

First, we are going to establish that we have $y \in N_{\mathbb{B}_{(l)}^{\mathcal{R}}}\left(\frac{x}{\|x\|_{(l)}^{\mathcal{R}}}\right)$, that is, the first of the two conditions in the characterization (16) of the subdifferential $\partial_{\dot{C}}\left(\varphi \circ \ell_{0}\right)(x)$.

On the one hand, because $\ell_{0}(y)=l$ and by $(18)$, we have that $\|y\|_{\star}=\|y\|_{(l), \star}^{\mathcal{R}}$. On the other hand, because $\ell_{0}(x)=l$ we have that $\|x\|=\|x\|_{(l)}^{\mathcal{R}}$ by $[4$, Equation (25a)]. Hence, from (17b), we get $\langle x, y\rangle=\|x\|_{(l)}^{\mathcal{R}} \times\|y\|_{(l), \star}^{\mathcal{R}}$, from which we obtain $y \in N_{\mathbb{B}_{(l)}^{\mathcal{R}}}\left(\frac{x}{\|x\|_{(l)}^{\mathcal{R}}}\right)$ by property (29) of the normal cone as $x \neq 0$. To close this part, notice that, for all $\lambda>0$, we have that $\lambda y \in N_{\mathbb{B}_{(l)}^{\mathcal{R}}}\left(\frac{x}{\|x\|_{(l)}^{\mathcal{R}}}\right)$, because this last set is a cone.

Second, we prove the other of the two conditions in the characterization (16) of the subdifferential $\partial_{\dot{C}}\left(\varphi \circ \ell_{0}\right)(x)$. More precisely, we are going to show that, for $\lambda$ large enough, $\|\lambda y\|_{(l), \star}^{\mathcal{R}}-\varphi(l)=$ $\sup _{j \in \llbracket 0, d \rrbracket}\left[\|\lambda y\|_{(j), \star}^{\mathcal{R}}-\varphi(j)\right]$. For this purpose, we consider the mapping $\left.\psi:\right] 0,+\infty[\rightarrow \mathbb{R}$ defined by

$$
\psi(\lambda)=\|\lambda y\|_{(l), \star}^{\mathcal{R}}-\varphi(l)-\sup _{j \in \llbracket 0, d \rrbracket}\left[\|\lambda y\|_{(j), \star}^{\mathcal{R}}-\varphi(j)\right], \quad \forall \lambda>0,
$$


and we will show that $\psi(\lambda)=0$ for $\lambda$ large enough. We have

$$
\begin{aligned}
& \psi(\lambda)= \inf _{j \in \llbracket 0, d \rrbracket}\left(\lambda\left(\|y\|_{(l), \star}^{\mathcal{R}}-\|y\|_{(j), \star}^{\mathcal{R}}\right)+\varphi(j)-\varphi(l)\right) \\
&= \inf \left\{\lambda\|y\|_{(l), \star}^{\mathcal{R}}+\varphi(0)-\varphi(l), \inf _{j \in \llbracket 1, l-1 \rrbracket}\left(\lambda\left(\|y\|_{(l), \star}^{\mathcal{R}}-\|y\|_{(j), \star}^{\mathcal{R}}\right)+\varphi(j)-\varphi(l)\right),\right. \\
&\left.\quad \inf _{j \in \llbracket l, d \rrbracket}\left(\lambda\left(\|y\|_{(l), \star}^{\mathcal{R}}-\|y\|_{(j), \star}^{\mathcal{R}}\right)+\varphi(j)-\varphi(l)\right)\right\} \\
&=\inf \left\{\lambda\|y\|_{(l), \star}^{\mathcal{R}}+\varphi(0)-\varphi(l), \inf _{j \in \llbracket 1, l-1 \rrbracket}^{\mathcal{R}}\left(\lambda\left(\|y\|_{(l), \star}^{\mathcal{R}}-\|y\|_{(j), \star}^{\mathcal{R}}\right)+\varphi(j)-\varphi(l)\right),\right. \\
&\left.\quad \inf _{j \in \llbracket l, d \rrbracket}(\varphi(j)-\varphi(l))\right\} \quad\left(\operatorname{as}\left\|_{(j)}^{\mathcal{R}}\right\|_{(j), \star}^{\mathcal{R}}=\|y\|_{(l), \star}^{\mathcal{R}} \text { for } j \geq l \text { by }(18)\right) \\
&=\inf \left\{\lambda\|y\|_{(l), \star}^{\mathcal{R}}+\varphi(0)-\varphi(l), \inf _{j \in \llbracket 1, l-1 \rrbracket}\left(\lambda\left(\|y\|_{(l), \star}^{\mathcal{R}}-\|y\|_{(j), \star}^{\mathcal{R}}\right)+\varphi(j)-\varphi(l)\right), 0\right\},
\end{aligned}
$$

$\operatorname{as}_{\inf _{j \in \llbracket l, d \rrbracket}}(\varphi(j)-\varphi(l))=0$ because $\varphi: \llbracket 0, d \rrbracket \rightarrow \mathbb{R}$ is a nondecreasing function. Let us show that the two first terms in the infimum go to $+\infty$ when $\lambda \rightarrow+\infty$. The first term $\lambda\|y\|_{(l), \star}^{\mathcal{R}}+$ $\varphi(0)-\varphi(l)$ goes to $+\infty$ because, by (18), we have $\|y\|_{(l), \star}^{\mathcal{R}}=\|y\|_{\star}>0$ as $y \in \mathbb{R}^{d} \backslash\{0\}$. The

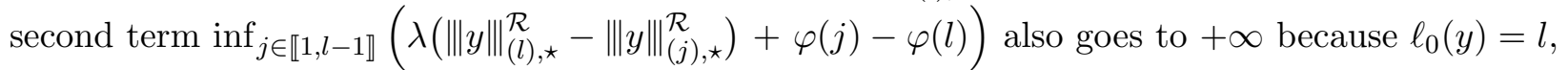
so that $\|y\|_{\star}=\|y\|_{(l), \star}^{\mathcal{R}}>\|y\|_{(j), \star}^{\mathcal{R}}$ for $j \in \llbracket 1, l-1 \rrbracket$ again by (18). Therefore, we deduce that $\psi(\lambda)=0$ for $\lambda$ large enough, and thus $\|\lambda y\|_{(l), \star}^{\mathcal{R}}-\varphi(l)=\sup _{j \in \llbracket 0, d \rrbracket}\left[\|\lambda y\|_{(j), \star}^{\mathcal{R}}-\varphi(j)\right]$, that is, $l \in \arg \max _{j \in \llbracket 0, d \rrbracket}\left[\|\lambda y\|_{(j), \star}^{\mathcal{R}}-\varphi(j)\right]$.

Wrapping up the above results, we have shown that, for any vector $y \in \mathbb{R}^{d}$ such that $\operatorname{supp}(y)=$ $\operatorname{supp}(x)$, and that $\langle x, y\rangle=\|x\| \times\|y\|_{\star}$, then, for $\lambda>0$ large enough, $\lambda y$ satisfies the two conditions in the characterization (16) of the subdifferential $\partial_{\dot{c}}\left(\varphi \circ \ell_{0}\right)(x)$. Hence, we get that $\lambda y \in \partial_{\dot{C}}\left(\varphi \circ \ell_{0}\right)(x)$.

This ends the proof.

Our proof of Proposition 7, hence of Theorem 6, uses the property that the nondecreasing function $\varphi: \llbracket 0, d \rrbracket \rightarrow \mathbb{R}$ takes finite values. What happens for $\varphi: \llbracket 0, d \rrbracket \rightarrow \overline{\mathbb{R}}$ ? Regarding CAPRA-convexity of a nondecreasing function of the $\ell_{0}$ pseudonorm taking infinite values, we suspect that a proof would rely on different assumptions than those of Proposition 7 and Theorem 6. As an indication, the comment after the proof of [4, Corollary 13] points out that, when the normed space $\left(\mathbb{R}^{d}, \mid\|\cdot \cdot\|\right)$ is strictly convex (that is, when the unit ball $\mathbb{B}$ is rotund), then $\delta_{\llbracket 0, k \rrbracket} \circ \ell_{0}$ is c-convex for $k \in \llbracket 0, d \rrbracket$. As the normed space $\left(\mathbb{R}^{d},\|\cdot\|_{p}\right)$, equipped with the $\ell_{p}$-norm $\|\cdot\|_{p}$ (for $p \in[1, \infty]$ ), is strictly convex if and only if $\left.p \in\right] 1$, $\infty[$, we get that the characteristic functions of the level sets of the $\ell_{0}$ pseudonorm are c-convex when the source norm $\|\cdot\|=\|\cdot\|_{p}$ for $\left.p \in\right] 1, \infty[$. 


\section{Convex factorization and variational formulation for the $\ell_{0}$ pseudonorm}

In this Section, we suppose that both the norm $|\|\cdot\|| \|$ and the dual norm $\|\cdot \mid\|_{\star}$ are orthantstrictly monotonic. In $\S 3.1$, we show that any nonnegative nondecreasing function of the pseudonorm $\ell_{0}$ coincides, on the unit sphere, with a proper convex lsc function on $\mathbb{R}^{d}$, and we provide various expressions for this latter function. In $\S 3.2$, we deduce a variational formula for nonnegative nondecreasing functions of the $\ell_{0}$ pseudonorm.

\subsection{Convex factorization and hidden convexity in the $\ell_{0}$ pseudo- norm}

We now present a (rather unexpected) consequence of the just established property that any nondecreasing function of the pseudonorm $\ell_{0}$ is CAPRA-convex (Theorem 6 ). Indeed, we prove that any nonnegative nondecreasing function of the pseudonorm $\ell_{0}$ coincides, on the unit sphere $\mathbb{S}=\left\{x \in \mathbb{R}^{d} \mid\|x\|=1\right\}$, with a proper convex lsc function on $\mathbb{R}^{d}$, and that this property extends to subdifferentials. We also provide various expressions for the underlying proper convex lsc function.

Proposition 8 Let $|\|\cdot\||$ be a norm on $\mathbb{R}^{d}$ with associated sequence $\left\{\mid\|\cdot\|_{\star,(j)}^{\text {tn }}\right\}_{j \in \llbracket 1, d \rrbracket}$ of general-


in Definition 3, and with associated CAPRA coupling $c$ as in (4). Suppose that both the norm $\|\cdot\|$ and the dual norm $\|\cdot\|_{\star}$ are orthant-strictly monotonic. Let $\varphi: \llbracket 0, d \rrbracket \rightarrow \mathbb{R}_{+}$be a nonnegative nondecreasing function, such that $\varphi(0)=0$. We define the function $\mathcal{L}_{0}^{\varphi}: \mathbb{R}^{d} \rightarrow \overline{\mathbb{R}}$ by

$$
\mathcal{L}_{0}^{\varphi}=\left(\left(\varphi \circ \ell_{0}\right)^{\phi}\right)^{\star^{\prime}}
$$

Then, the following statements hold true.

(a) The function $\mathcal{L}_{0}^{\varphi}: \mathbb{R}^{d} \rightarrow \overline{\mathbb{R}}$ is proper convex lsc.

(b) The function $\varphi \circ \ell_{0}$ coincides, on the unit sphere $\mathbb{S}=\left\{x \in \mathbb{R}^{d} \mid\|x\|=1\right\}$, with the function $\mathcal{L}_{0}^{\varphi}$, that is,

$$
\left(\varphi \circ \ell_{0}\right)(s)=\mathcal{L}_{0}^{\varphi}(s), \forall s \in \mathbb{S}
$$

(c) The CAPRA-subdifferential, as in (13), of the function $\varphi \circ \ell_{0}$ coincides, on the unit sphere $\mathbb{S}$, with the (Rockafellar-Moreau) subdifferential', as in (35a), of the function $\mathcal{L}_{0}^{\varphi}$, that is,

$$
\partial_{C^{\prime}}\left(\varphi \circ \ell_{0}\right)(s)=\partial \mathcal{L}_{0}^{\varphi}(s), \forall s \in \mathbb{S}
$$

\footnotetext{
${ }^{7}$ We can also use the definition (35b) of the (Rockafellar-Moreau) subdifferential as the function $\mathcal{L}_{0}^{\varphi}$ is proper convex and as $\mathbb{S} \subset \operatorname{dom} \mathcal{L}_{0}^{\varphi}$ by $\left(20\right.$ a) since $\varphi: \llbracket 0, d \rrbracket \rightarrow \mathbb{R}_{+}$takes finite values.
} 
(d) Convex factorization property. The function $\varphi \circ \ell_{0}$ can be expressed as the composition of the proper convex lsc function $\mathcal{L}_{0}^{\varphi}$ with the normalization mapping $n$ in (5), that is,

$$
\varphi \circ \ell_{0}=\mathcal{L}_{0}^{\varphi} \circ n
$$

or, equivalently,

$$
\left(\varphi \circ \ell_{0}\right)(x)=\mathcal{L}_{0}^{\varphi}\left(\frac{x}{\|x\| \|}\right), \forall x \in \mathbb{R}^{d} \backslash\{0\}
$$

(e) The function $\mathcal{L}_{0}^{\varphi}$ is given by

$$
\mathcal{L}_{0}^{\varphi}=\left(\sup _{j \in \llbracket 0, d \rrbracket}\left[\|\cdot\|_{\star,(j)}^{\mathrm{tn}}-\varphi(j)\right]\right)^{\star^{\prime}} .
$$

(f) The function $\mathcal{L}_{0}^{\varphi}$ is the largest convex lsc function below the integer valued function

$$
\mathbb{R}^{d} \ni x \mapsto \inf _{j \in \llbracket 0, d \rrbracket}\left[\delta_{\mathbb{B}_{\star}^{\star s n}(j)}(x)+\varphi(j)\right]
$$

that is, below the function $x \in \mathbb{B}_{\star,(j)}^{\star \mathrm{sn}} \backslash \mathbb{B}_{\star,(j-1)}^{\star \mathrm{sn}} \mapsto \varphi(j)$ for $j \in \llbracket 1, d \rrbracket$ and $x \in \mathbb{B}_{\star,(0)}^{\star \mathrm{sn}}=$ $\{0\} \mapsto 0$, the function being infinite outside $\mathbb{B}_{\star,(d)}^{\star \mathrm{sn}}=\mathbb{B}$ (the above construction makes sense as $\left.\mathbb{B}_{\star,(1)}^{\star \mathrm{sn}} \subset \cdots \subset \mathbb{B}_{\star,(j-1)}^{\star \mathrm{sn}} \subset \mathbb{B}_{\star,(j)}^{\star \mathrm{sn}} \subset \cdots \subset \mathbb{B}_{\star,(d)}^{\star s \mathrm{sn}}=\mathbb{B}\right)$.

(g) The function $\mathcal{L}_{0}^{\varphi}$ is the largest convex lsc function below the integer valued function

$$
\mathbb{R}^{d} \ni x \mapsto \inf _{j \in \llbracket 0, d \rrbracket}\left[\delta_{\mathbb{S}_{\star}^{\star s \mathrm{sn}}}(x)+\varphi(j)\right]
$$

that is, below the function $x \in \mathbb{R}^{d} \mapsto \inf \varphi\left\{j \in\{0, \ldots, d\} \mid x \in \mathbb{S}_{\star,(j)}^{\star \mathrm{sn}}\right\}$, with the convention that $\mathbb{S}_{\star,(0)}^{\star \mathrm{sn}}=\{0\}$ and that $\inf \emptyset=+\infty$.

(h) The proper convex lsc function $\mathcal{L}_{0}^{\varphi}$ also has three variational expressions as follows, where $\Delta_{d+1}$ is the simplex of $\mathbb{R}^{d+1}$,

$$
\begin{aligned}
\mathcal{L}_{0}^{\varphi}(x)= & \min _{\substack{\left(\lambda_{0}, \lambda_{1}, \ldots, \lambda_{d}\right) \in \Delta_{d+1} \\
x \in \sum_{l=1}^{d} \lambda_{j} \mathbb{B}_{\star,(l)}^{\star \mathrm{sn}}}} \sum_{l=1}^{d} \lambda_{j} \varphi(j), \quad \forall x \in \mathbb{R}^{d} \\
= & \min _{\substack{\left(\lambda_{0}, \lambda_{1}, \ldots, \lambda_{d}\right) \in \Delta_{d+1} \\
x \in \sum_{l=1}^{d} \lambda_{j} \mathbb{S}_{\star,(l)}^{\star \mathrm{sn}}}} \sum_{l=1}^{d} \lambda_{j} \varphi(j), \quad \forall x \in \mathbb{R}^{d} \\
= & \min _{\substack{x^{(1)} \in \mathbb{R}^{d}, \ldots, x^{(d)} \in \mathbb{R}^{d} \\
\sum_{j=1}^{d}\left\|x^{(j)}\right\|_{\star,,(j)}^{\text {sn }} \leq 1 \\
\sum_{j=1}^{d} x^{(j)}=x}} \sum_{j=1}^{d} \varphi(j)\left\|x^{(j)}\right\|_{\star,(j)}^{\star \mathrm{sn}}, \quad \forall x \in \mathbb{R}^{d} .
\end{aligned}
$$


Proof. As in the beginning of the proof of Proposition 7, we make the observation that, since the norm $\|\cdot\| \cdot \|$ is orthant-strictly monotonic, it is orthant-monotonic, so that we have $\|\cdot\| \cdot\left\|_{(j)}^{\mathcal{R}}=\right\| \cdot\|\cdot\|_{\star,(j)}^{\star \mathrm{sn}}$ and $\|\cdot\|_{(j), \star}^{\mathcal{R}}=\|\| \cdot \|_{\star,(j)}^{\text {tn }}$, for $j \in \llbracket 0, d \rrbracket$ by (31) in Proposition 13 (with the convention that these are the null seminorms in the case $j=0)$.

(a) As the Fenchel conjugacy induces a one-to-one correspondence between the closed convex functions on $\mathbb{R}^{d}$ and themselves [18, Theorem 5], the function $\mathcal{L}_{0}^{\varphi}=\left(\left(\varphi \circ \ell_{0}\right)^{\boldsymbol{c}}\right)^{\star^{\prime}}$ in $(19)$ is closed convex. We now show that it is proper. Indeed, on the one hand, it is easily seen by the very definition $(6 \mathrm{a})$ that the function $\left(\varphi \circ \ell_{0}\right)$ c takes finite values, from which we deduce that the function $\left(\left(\varphi \circ \ell_{0}\right)^{\phi}\right)^{\star^{\prime}}$ never takes the value $-\infty$, by $(34 \mathrm{~b})$. On the other hand, we have $\left(\varphi \circ \ell_{0}\right)^{\dot{c} c^{\prime}}=$ $\left(\left(\varphi \circ \ell_{0}\right)^{c}\right)^{\star^{\prime}} \circ n$ by [4, Equation (30d)], and $\left(\varphi \circ \ell_{0}\right)^{c c^{\prime}} \leq \varphi \circ \ell_{0}$ by $(6 \mathrm{c})$, from which we deduce that, for any $x \in \mathbb{S}$, we have $\left(\left(\varphi \circ \ell_{0}\right)^{\dot{c}}\right)^{\star^{\prime}}(x)=\left(\varphi \circ \ell_{0}\right)^{\dot{c} \dot{C}^{\prime}}(x) \leq\left(\varphi \circ \ell_{0}\right)(x)<+\infty$ since $\varphi: \llbracket 0, d \rrbracket \rightarrow \mathbb{R}_{+}$.

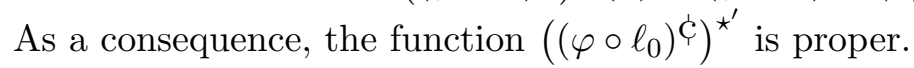

(b) The assumptions make it possible to conclude that $\left(\varphi \circ \ell_{0}\right)^{\phi \dot{c}^{\prime}}=\varphi \circ \ell_{0}$, thanks to Theorem 6 . We deduce from [4, Proposition 10] that, being c-convex, the function $\varphi \circ \ell_{0}$ coincides, on the unit sphere $\mathbb{S}$, with the closed convex function $\mathcal{L}_{0}^{\varphi}: \mathbb{R}^{d} \rightarrow \overline{\mathbb{R}}$ given by [4, Equation (30d)] namely $\mathcal{L}_{0}^{\varphi}=\left(\left(\varphi \circ \ell_{0}\right)^{\dot{c}}\right)^{\star^{\prime}}$. Thus, we have proved (20a).

(c) Let $s \in \mathbb{S}$. We prove $(20 \mathrm{~b})$ as follows:

$$
y \in \partial \mathcal{L}_{0}^{\varphi}(s) \Longleftrightarrow\left(\mathcal{L}_{0}^{\varphi}\right)^{\star}(y)=\langle s, y\rangle+\left(-\mathcal{L}_{0}^{\varphi}(s)\right)
$$

by definition (35a) of the (Rockafellar-Moreau) subdifferential of a function

$$
\begin{aligned}
& \Longleftrightarrow\left(\left(\left(\varphi \circ \ell_{0}\right)^{\dot{c}}\right)^{\star^{\prime}}\right)^{\star}(y)=\langle s, y\rangle+\left(-\left(\left(\left(\varphi \circ \ell_{0}\right)^{\dot{c}}\right)^{\star^{\prime}}\right)(s)\right) \\
& \left.\quad \text { (by definition }(19) \text { of } \mathcal{L}_{0}^{\varphi}=\left(\left(\varphi \circ \ell_{0}\right)^{\dot{c}}\right)^{\star^{\prime}}\right) \\
& \Longleftrightarrow\left(\varphi \circ \ell_{0}\right)^{\dot{C}}(y)=\langle s, y\rangle+\left(-\left(\left(\left(\varphi \circ \ell_{0}\right)^{\dot{c}}\right)^{\star^{\prime}}\right)(s)\right)
\end{aligned}
$$

because the function $\left(\varphi \circ \ell_{0}\right)^{c}$ is a Fenchel conjugate by [4, Equation (30b)], hence is closed convex, hence is equal to its Fenchel biconjugate $\left(\left(\left(\varphi \circ \ell_{0}\right)^{\dot{c}}\right)^{\star^{\prime}}\right)^{\star}$

$$
\begin{aligned}
& \Longleftrightarrow\left(\varphi \circ \ell_{0}\right)^{\dot{c}}(y)=c(s, y)+\left(-\left(\left(\left(\varphi \circ \ell_{0}\right)^{\dot{c}}\right)^{\star^{\prime}}\right)(s)\right) \\
& \text { (by definition }(4) \text { of } \phi(s, y) \text { as } s \in \mathbb{S}) \\
& \Longleftrightarrow\left(\varphi \circ \ell_{0}\right)^{\dot{c}}(y)=\dot{c}(s, y)+\left(-\left(\left(\varphi \circ \ell_{0}\right)^{\dot{c} \dot{c}^{\prime}}\right)(s)\right)
\end{aligned}
$$

because $\left(\varphi \circ \ell_{0}\right)^{\dot{c} \dot{c}^{\prime}}=\left(\left(\varphi \circ \ell_{0}\right)^{\dot{c}}\right)^{\star^{\prime}} \circ n$ by $[4$, Equation $(30 \mathrm{~d})]$, and using that $n(s)=s$ since $s \in \mathbb{S}$ by definition (5) of the normalization mapping $n$

$$
\begin{aligned}
& \Longleftrightarrow\left(\varphi \circ \ell_{0}\right)^{\dot{\phi}}(y)=\dot{c}(s, y)+\left(-\left(\varphi \circ \ell_{0}\right)(s)\right) \quad\left(\text { as }\left(\varphi \circ \ell_{0}\right)^{c c^{\prime}}=\varphi \circ \ell_{0} \text { by Theorem } 6\right) \\
& \Longleftrightarrow y \in \partial_{\dot{C}}\left(\varphi \circ \ell_{0}\right)(s) . \quad \text { (by definition }(13) \text { of the CAPRA-subdifferential) }
\end{aligned}
$$


(d) The equality (20c) is a consequence of the formula $\varphi \circ \ell_{0}=\left(\varphi \circ \ell_{0}\right)^{\phi} \dot{c}^{\prime}=\left(\left(\varphi \circ \ell_{0}\right)^{\dot{c}}\right)^{\star^{\prime}} \circ n$ given by [4, Equation (30d)]. The equality (20d) is an easy consequence of (20c) and of the definition (5) of the normalization mapping $n$.

(e) As $\mathcal{L}_{0}^{\varphi}=\left(\left(\varphi \circ \ell_{0}\right)^{\dot{\varphi}}\right)^{\star^{\prime}}$ by definition $(19)$, and as we have that $\left(\left(\varphi \circ \ell_{0}\right)^{\dot{c}}\right)^{\star^{\prime}}=\left(\sup _{j \in \llbracket 0, d \rrbracket}\left[\|\cdot\| \|_{\star,(j)}^{\text {tn }}-\varphi(j)\right]\right)^{\star^{\prime}}$ by (14), we get (21a).

(f) We use [4, Proposition 12], and especially Equations (34c) and (34d), to obtain (21b). The


generalized coordinate- $k$ norms (see Definition 2) in $[4$, Equation $(24)]$. Now, since $\|\cdot \cdot\|_{(j)}^{\mathcal{R}}=\|\cdot\|_{\star,(j)}^{\star s n}$, as noticed at the beginning of the proof, we get that $\mathbb{B}_{\star,(1)}^{\star \mathrm{sn}} \subset \cdots \subset \mathbb{B}_{\star,(j-1)}^{\star \mathrm{sn}} \subset \mathbb{B}_{\star,(j)}^{\star \mathrm{sn}} \subset \cdots \subset \mathbb{B}_{\star,(d)}^{\star \mathrm{sn}}=$ $\mathbb{B}$.

(g) We use [4, Proposition 12], and especially Equations (34e) and (34f), to obtain (21c).

(h) We use [4, Proposition 12], and especially Equations (34g), (34h) and (34i) to obtain (22a), $(22 \mathrm{~b})$ and $(22 \mathrm{c})$.

This ends the proof.

\subsection{Variational formulation for the $\ell_{0}$ pseudonorm}

As an application of Proposition 8, we obtain the second main result of this paper, namely a variational formulation for (nonnegative nondecreasing functions of) the $\ell_{0}$ pseudonorm.

Theorem 9 Let $\|\cdot\| \cdot \|$ be a norm on $\mathbb{R}^{d}$ with associated sequence $\left\{\|\cdot\| \|_{\star,(j)}^{\text {tn }}\right\}_{j \in \llbracket 1, d \rrbracket}$ of generalized k-support dual norms as in Definition 3, and with associated CAPRA coupling $c$ as in (4). Suppose that both the norm $\|\cdot\| \cdot \|$ and the dual norm $\|\cdot\|_{\star}$ are orthant-strictly monotonic. Let $\varphi: \llbracket 0, d \rrbracket \rightarrow \mathbb{R}_{+}$be a nonnegative nondecreasing function such that $\varphi(0)=0$. Then, we have the equality

$$
\varphi\left(\ell_{0}(x)\right)=\frac{1}{\|x\|} \min _{\substack{x^{(1)} \in \mathbb{R}^{d}, \ldots, x^{(d)} \in \mathbb{R}^{d} \\ \sum_{j=1}^{d}\left\|x^{(j)}\right\|_{\star,(j)}^{\star s n} \leq\|x\| \\ \sum_{j=1}^{d} x^{(j)}=x}} \sum_{j=1}^{d} \varphi(j)\left\|x^{(j)}\right\|_{\star,(j)}^{\star \mathrm{sn}}, \forall x \in \mathbb{R}^{d} \backslash\{0\}
$$

where the sequence of generalized $k$-support dual norms $\left\{\|\cdot\|_{\star,(j)}^{\star s n}\right\}_{j \in \llbracket 1, d \rrbracket}$ has been introduced in Definition 3.

When $\ell_{0}(x)=l \geq 1$, the minimum in $(23)$ is achieved at $\left(x^{(1)}, \ldots, x^{(d)}\right) \in\left(\mathbb{R}^{d}\right)^{d}$ such that $x^{(j)}=0$ for $j \neq l$ and $x^{(l)}=x$.

Proof. Equation (23) derives from (20c) where we use the expression (22c) for the function $\mathcal{L}_{0}^{\varphi}$ in (19). 
Now for the argmin. When $\ell_{0}(x)=l \geq 1$, we have that $\|x\|=\|x\|_{(d)}^{\mathcal{R}}=\ldots=\|x\|_{(l)}^{\mathcal{R}}$ by $[4$, Equation (25a)]. Now, for any $k \in \llbracket 1, d \rrbracket$, we have $\|\cdot\|_{(k)}^{\mathcal{R}}=\|\cdot \cdot\|_{\star,(k)}^{\star \mathrm{sn}}$ by (31) in Proposition 13 , since the norm $\|\cdot \cdot\|$ is orthant-strictly monotonic, hence is orthant-monotonic. As a consequence, we have that $\|x\|=\|x\|_{\star,(d)}^{\star \mathrm{sn}}=\ldots=\|x\|_{\star,(l)}^{\star \mathrm{sn}}$. Therefore, the vectors $x^{(1)} \in \mathbb{R}^{d}, \ldots, x^{(d)} \in \mathbb{R}^{d}$ defined by $x^{(j)}=0$ for $j \neq l$ and $x^{(l)}=x$ are admissible for the minimization problem (23). We deduce from $(23)$ that $\varphi(l)=\varphi\left(\ell_{0}(x)\right) \leq \frac{1}{\|x\|} \varphi(l)\|x\|_{\star,(l)}^{\star \mathrm{sn}}=\varphi(l)$.

This ends the proof.

As an illustration, Theorem 9 applies when the norm $\|\cdot\| \cdot \|$ is any of the $\ell_{p}$-norms $\|\cdot\|_{p}$ on the space $\mathbb{R}^{d}$, for $\left.p \in\right] 1, \infty[$, and Equation (23) then gives (see the notations in the second column of Table 1)

$$
\begin{array}{r}
\left(\varphi \circ \ell_{0}\right)(x)=\frac{1}{\|x\|_{p}} \min _{\substack{x^{(1)} \in \mathbb{R}^{d}, \ldots, x^{(d)} \in \mathbb{R}^{d} \\
\sum_{j=1}^{d}\left\|x^{(j)}\right\|_{p, j}^{\mathrm{sn}} \leq\|x\|_{p} \\
\sum_{j=1}^{d} x^{(j)}=x}} \sum_{j=1}^{d} \varphi(j)\left\|x^{(j)}\right\|_{p, j}^{\mathrm{sn}}, \\
\left.\forall x \in \mathbb{R}^{d} \backslash\{0\}, \forall p \in\right] 1, \infty[.
\end{array}
$$

Indeed, when $p \in] 1, \infty\left[\right.$, the $\ell_{p}$-norm $\|\cdot\|\|=\| \cdot \|_{p}$ is orthant-strictly monotonic, and so is its dual norm $\|\cdot\|_{\star}=\|\cdot\|_{q}$ where $1 / p+1 / q=1$. When $p=1$, the $\ell_{1}$-norm $\|\cdot\|=\|\cdot\|_{1}$ is orthant-strictly monotonic, but the dual norm $\|\cdot\|\|=\| \cdot \|_{\infty}$ is not; when $p=\infty$, the $\ell_{\infty}$-norm $\|\cdot \mid\|=\|\cdot\|_{\infty}$ is not orthant-strictly monotonic; hence, in those two extreme cases, we cannot conclude (but we obtain inequalities like in [4, Equation (25a)]).

Finally, with the novel expression (23) for the $\ell_{0}$ pseudonorm, we deduce a possible reformulation of exact sparse optimization problems as follows (the proof is a straightforward application of Theorem 9).

Proposition 10 Let $C \subset \mathbb{R}^{d}$ be such that $0 \notin C$. Let $\|\cdot\|$ be a norm on $\mathbb{R}^{d}$, such that both the norm $\|\mid\| \cdot \|$ and the dual norm $\|\cdot\| \|_{\star}$ are orthant-strictly monotonic. Let $\varphi: \llbracket 0, d \rrbracket \rightarrow \mathbb{R}_{+}$

\footnotetext{
${ }^{8}$ If we had $0 \in C$, the minimization problem below would be obviously achieved at $x=0$.
} 
be a nondecreasing function, such that $\varphi(0)=0$. Then, we have:

$$
\begin{aligned}
& \min _{x \in C} \varphi\left(\ell_{0}(x)\right)=\min _{\substack{x \in C, x^{(1)} \in \mathbb{R}^{d}, \ldots, x^{(d)} \in \mathbb{R}^{d} \\
\sum_{j=1}^{d}\left\|x^{(j)}\right\|_{*,(j)}^{* n} \leq 1 \\
\sum_{j=1}^{d} x^{(j)}=\frac{x}{\|x\|}}} \sum_{j=1}^{d} \varphi(j)\left\|x^{(j)}\right\|_{\star,(j)}^{\star \mathrm{sn}},
\end{aligned}
$$



$$
\begin{aligned}
& \sum_{j=1}^{d}\left\|x^{(j)}\right\|_{\star,(j)}^{\star \star n n} \leq\|x\| \\
& \sum_{j=1}^{d} x^{(j)}=x \\
& =\min _{x \in C} \frac{1}{\|x\|} \min _{\substack{x^{(1)} \in \mathbb{R}^{d}, \ldots, x^{(d)} \in \mathbb{R}^{d} \\
\sum_{j=1}^{d}\left\|x^{(j)}\right\|_{*,(j)}^{\mathrm{sn}} \leq\|x\|}} \sum_{j=1}^{d} \varphi(j)\left\|x^{(j)}\right\|_{\star,(j)}^{\star \mathrm{sn}} . \\
& \sum_{j=1}^{d} x^{(j)}=x \\
& \text { convex optimization problem }
\end{aligned}
$$

\section{Conclusion}

In this paper, we have proven that the $\ell_{0}$ pseudonorm is equal to its CAPRA-biconjugate when both the source norm and its dual norm are orthant-strictly monotonic. In that case, one says that the $\ell_{0}$ pseudonorm is a CAPRA-convex function. A surprising consequence is the convex factorization property, a way to express hidden convexity: the $\ell_{0}$ pseudonorm coincides, on the unit sphere of the source norm, with a proper convex lsc function. More generally, this holds true for any function of the $\ell_{0}$ pseudonorm that is nondecreasing, with finite values. Then, we have obtained exact variational formulations for the $\ell_{0}$ pseudonorm, suitable for exact sparse optimization. For this purpose, we have introduced sequences of generalized top- $k$ and $k$-support dual norms. We now briefly perspectives for exact sparse optimization.

The reformulations for exact sparse optimization problems, obtained in Proposition 10, have the nice feature to display partial convexity but make use of new (latent) vectors, in same number than the underlying dimension $d$. Thus, the algorithmic implementation may be delicate. However, the variational formulation obtained may suggest approximations of the $\ell_{0}$ pseudonorm, involving generalized $k$-support dual norms, which, themselves, may lead to new smooth sparsity inducing terms. Finally, we have identified elements of the CAPRAsubdifferential of nondecreasing functions of the $\ell_{0}$ pseudonorm, and we have related this CAPRA-subdifferential with the Rockafellar-Moreau subdifferential of the associated convex lsc function (in the convex factorization property). The identification of such subgradients could inspire "gradient-like" algorithms.

Acknowledgements. We thank Guillaume Obozinski for discussions on first versions of this work, and Jean-Baptiste Hiriart-Urruty for his comments. We are indebted to two 
Reviewers and to the Editor who, by their questions and comments, helped us improve the manuscript.

\section{A Properties of relevant norms for the $\ell_{0}$ pseudonorm}

We provide background on properties of norms that prove relevant for the $\ell_{0}$ pseudonorm. In $\S$ A.1, we review notions related to dual norms. We establish properties of orthantmonotonic and orthant-strictly monotonic norms in $\S \mathrm{A} .2$, and of coordinate- $k$ and dual coordinate- $k$ norms in $\S$ A.3.

\section{A.1 Dual norm, $\|\cdot\| \cdot$-duality, normal cone}

For any norm $\|\cdot \mid\|$ on $\mathbb{R}^{d}$, we recall that the following expression

$$
\|y\|_{\star}=\sup _{\|x\| \leq 1}\langle x, y\rangle, \forall y \in \mathbb{R}^{d}
$$

defines a norm on $\mathbb{R}^{d}$, called the dual norm $\|\cdot\|_{\star}$ (in $[20$, Section 15$]$, this operation is widened to a polarity operation between closed gauges).

By definition of the dual norm in (26), we have the inequality

$$
\langle x, y\rangle \leq\|x\|\|\times\| y \|_{\star}, \quad \forall(x, y) \in \mathbb{R}^{d} \times \mathbb{R}^{d} .
$$

We are interested in the case where this inequality is an equality. One says that $y \in \mathbb{R}^{d}$ is $\|\cdot\| \cdot \|$-dual to $x \in \mathbb{R}^{d}$, denoted by $y \|_{\|\cdot\|} x$, if equality holds in inequality (27a), that is,

$$
y\left\|_{\|\cdot\|} x \Longleftrightarrow\langle x, y\rangle=\right\|\|x\| \times\|\| y \|_{\star} .
$$

The terminology $\|\mid\| \cdot \|$-dual comes from [11, page 2] (see also the vocable of dual vector pair in [7, Equation (1.11)] and of dual vectors in [8, p. 283], whereas it is refered as polar alignment in [6]). It will be convenient to express this notion of $\|\cdot\| \cdot \|$-duality in terms of geometric objects of convex analysis. For this purpose, we recall that the normal cone $N_{C}(x)$ to the (nonempty) closed convex subset $C \subset \mathbb{R}^{d}$ at $x \in C$ is the closed convex cone defined by [10, p.136]

$$
N_{C}(x)=\left\{y \in \mathbb{R}^{d} \mid\left\langle y, x^{\prime}-x\right\rangle \leq 0, \forall x^{\prime} \in C\right\} .
$$

Now, an easy computation shows that the notion of $\|\cdot \mid\|$-duality can be rewritten in terms of normal cone $N_{\mathbb{B}}$ as follows:

$$
y \|_{\|\cdot\|} x \Longleftrightarrow y \in N_{\mathbb{B}}\left(\frac{x}{\|x\|}\right), \forall(x, y) \in \mathbb{R}^{d} \backslash\{0\} \times \mathbb{R}^{d} .
$$




\section{A.2 Properties of orthant-strictly monotonic norms}

We provide useful properties of orthant-monotonic and orthant-strictly monotonic norms (see Definition 5). We recall that $x_{K} \in \mathcal{R}_{K}$ denotes the vector which coincides with $x$, except for the components outside of $K$ that vanish, and that the subspace $\mathcal{R}_{K}$ of $\mathbb{R}^{d}$ has been defined in (7).

Proposition 11 Let $\|\cdot\| \|$ be an orthant-monotonic norm on $\mathbb{R}^{d}$. Then, the dual norm $\|\cdot\| \|_{\star}$ is orthant-monotonic, and the norm $\|\cdot\| \|$ is increasing with the coordinate subspaces, in the sense that, for any $x \in \mathbb{R}^{d}$ and any $J \subset K \subset \llbracket 1, d \rrbracket$, we have $\left\|x_{J}\right\| \leq\left\|x_{K}\right\| \|$.

Proof. Let $\||\cdot|\|$ be an orthant-monotonic norm on $\mathbb{R}^{d}$. Then, by [7, Theorem 2.23], the dual norm $\|\cdot\|_{\star}$ is also orthant-monotonic and, by [11, Proposition 2.4], we have that $\|u\| \leq\|u+v\|$, for any vectors $u \in \mathcal{R}_{J}$ and $^{9} v \in \mathcal{R}_{-J}$, and for any subset $J \subset \llbracket 1, d \rrbracket$. We consider $x \in \mathbb{R}^{d}$ and $J \subset K \subset \llbracket 1, d \rrbracket$. By setting $u=x_{J} \in \mathcal{R}_{J}$ and $v=x_{K}-x_{J}$, we get that $v \in \mathcal{R}_{-J}$, hence that $\left\|x_{J}\right\| \leq\left\|x_{K}\right\|$.

Proposition 12 Let $\|\cdot \mid\|$ be an orthant-strictly monotonic norm on $\mathbb{R}^{d}$. Then

(a) the norm $\|\cdot\| \mid \|$ is strictly increasing with the coordinate subspaces in the sense that, for any $x \in \mathbb{R}^{d}$ and any $J \subsetneq K \subset \llbracket 1, d \rrbracket$, we have $x_{J} \neq x_{K} \Rightarrow\left\|x_{J}\right\|<\left\|x_{K}\right\|$.

(b) for any vector $u \in \mathbb{R}^{d} \backslash\{0\}$, there exists a vector $v \in \mathbb{R}^{d} \backslash\{0\}$ such that $\operatorname{supp}(v)=$ $\operatorname{supp}(u)$, that $u \circ v \geq 0$, and that $v$ is $\|\cdot \mid\|-$ dual to $u$, that is, $\langle u, v\rangle=\|u\| \times\|v\| \|_{\star}$.

\section{Proof.}

(a) Let $x \in \mathbb{R}^{d}$ and $J \subsetneq K \subset \llbracket 1, d \rrbracket$ be such that $x_{J} \neq x_{K}$. We will show that $\left\|x_{K}\right\|>\left\|x_{J}\right\|$.

For this purpose, we set $u=x_{J}$ and $v=x_{K}-x_{J}$. Thus, we get that $u \in \mathcal{R}_{K}$ and $v \in \mathcal{R}_{-K} \backslash\{0\}$ (since $J \subsetneq K$ and $x_{J} \neq x_{K}$ ), that is, $u=u_{K}$ and $v=v_{-K} \neq 0$. We are going to show that $\|u+v\|>\|u\|$.

On the one hand, by definition of the module of a vector, we easily see that $|w|=\left|w_{K}\right|+\left|w_{-K}\right|$, for any vector $w \in \mathbb{R}^{d}$. Thus, we have $|u+v|=\left|(u+v)_{K}\right|+\left|(u+v)_{-K}\right|=\left|u_{K}+v_{K}\right|+\left|u_{-K}+v_{-K}\right|=$ $\left|u_{K}+0\right|+\left|0+v_{-K}\right|=\left|u_{K}\right|+\left|v_{-K}\right|>\left|u_{K}\right|=|u|$ since $\left|v_{-K}\right|>0$ as $v=v_{-K} \neq 0$, and since $u=u_{K}$. On the other hand, we easily get that $(u+v) \circ u=\left((u+v)_{K} \circ u_{K}\right)+\left((u+v)_{-K} \circ u_{-K}\right)=$ $\left(\begin{array}{lll}u_{K} \circ u_{K}\end{array}\right)+\left(v_{-K} \circ u_{-K}\right)=\left(\begin{array}{lll}u_{K} \circ u_{K}\end{array}\right)$, because $u_{-K}=0$. Therefore, we get that $(u+v) \circ u=$ $\left(u_{K} \circ u_{K}\right) \geq 0$.

From $|u+v|>|u|$ and $(u+v) \circ u \geq 0$, we deduce that $\|u+v\|>\|u\|$ by Definition 5 as the norm $\|\cdot\|$ is orthant-strictly monotonic. Since $u=x_{J}$ and $v=x_{K}-x_{J}$, we conclude that $\left\|x_{K}\right\|>\left\|x_{J}\right\|$.

(b) Let $u \in \mathbb{R}^{d} \backslash\{0\}$ be given and let us put $K=\operatorname{supp}(u) \neq \emptyset$. As the norm $\|\cdot\|$ is orthantstrictly monotonic, it is orthant-monotonic; hence, by [11, Proposition 2.4], there exists a vector

\footnotetext{
${ }^{9}$ Here, following notation from game theory, we have denoted by $-J$ the complementary subset of $J$ in $\llbracket 1, d \rrbracket: J \cup(-J)=\llbracket 1, d \rrbracket$ and $J \cap(-J)=\emptyset$.
} 
$v \in \mathbb{R}^{d} \backslash\{0\} \operatorname{such}$ that $\operatorname{supp}(v) \subset \operatorname{supp}(u)$, that $u \circ v \geq 0$ and that $v$ is $\|\cdot\| \cdot \|$-dual to $u$, as in (27b), that is, $\langle u, v\rangle=\|u\| \times\|v\|_{\star}$. Thus $J=\operatorname{supp}(v) \subset K=\operatorname{supp}(u)$. We will now show that $J \subsetneq K$ is impossible, hence that $J=K$, thus proving that Item $(\boldsymbol{b})$ holds true with the above vector $v$.

Writing that $\langle u, v\rangle=\|u\| \times\|v\|_{\star}$ (using that $u=u_{K}$ and $v=v_{K}=v_{J}$ ), we obtain

$$
\|u\| \times\|v\|_{\star}=\langle u, v\rangle=\left\langle u_{K}, v\right\rangle=\left\langle u_{K}, v_{K}\right\rangle=\left\langle u_{K}, v_{J}\right\rangle=\left\langle u_{J}, v_{J}\right\rangle=\left\langle u_{J}, v\right\rangle,
$$

by obvious properties of the scalar product $\langle\cdot, \cdot\rangle$. As a consequence, we get that $\left\{u_{K}, u_{J}\right\} \subset$ $\arg \max _{\|x\| \leq\|u\|}\langle x, v\rangle$, by definition (26) of $\|v\|_{\star}$, because $\|u\|=\left\|u_{K}\right\|\|\geq\| u_{J}\|\|$, by Proposition 11 since $J \subset K$ and the norm $\|\cdot\| \|$ is orthant-monotonic. But any solution in $\arg \max _{\|x\| \leq\|u\|}\langle x, v\rangle$ belongs to the frontier of the ball of radius $\|u\|$, hence has exactly norm $\|u\|$. Thus, we deduce that $\|u\|=\left\|u_{K}\right\|=\left\|u_{J}\right\|$. If we had $J=\operatorname{supp}(v) \subsetneq K=\operatorname{supp}(u)$, we would have $u_{J} \neq u_{K}$, hence $\left\|u_{K}\right\|\|\| u_{J} \|$ by Item $(\boldsymbol{a})$; this would be in contradiction with $\left\|u_{K}\right\|=\left\|u_{J}\right\|$. Therefore, $J=\operatorname{supp}(v)=K=\operatorname{supp}(u)$.

This ends the proof.

\section{A.3 Properties of coordinate- $k$ and dual coordinate- $k$ norms, and of generalized top- $k$ and $k$-support dual norms}

We establish useful properties of coordinate- $k$ and dual coordinate- $k$ norms (Definition 2), and of generalized top- $k$ and $k$-support dual norms (Definition 3 ).

Proposition 13 Let $\|\cdot\|$ be a source norm on $\mathbb{R}^{d}$.

Coordinate-k norms are greater than k-support dual norms, that is,

$$
\|x\|_{(k)}^{\mathcal{R}} \geq\|x\|_{\star,(k)}^{\star \mathrm{sn}}, \quad \forall x \in \mathbb{R}^{d}, \quad \forall k \in \llbracket 1, d \rrbracket,
$$

whereas dual coordinate- $k$ norms are lower than generalized top- $k$ dual norms, that is,

$$
\|y\|_{(k), \star}^{\mathcal{R}} \leq\|y\|_{\star,(k)}^{\text {tn }}, \forall y \in \mathbb{R}^{d}, \quad \forall k \in \llbracket 1, d \rrbracket .
$$

If the source norm norm $\|\cdot\| \mid \|$ is orthant-monotonic, then equalities hold true, that is,

$$
\|\cdot\| \text { is orthant-monotonic } \Rightarrow \forall k \in \llbracket 1, d \rrbracket\left\{\begin{array}{l}
\|\cdot\|_{(k)}^{\mathcal{R}}=\|\|_{*}^{*} \|_{\star,(k)}^{\star s n} \\
\|\| \cdot\left\|_{(k), \star}^{\mathcal{R}}=\right\| \cdot\|\|_{\star,(k)}^{\text {tn }}
\end{array} .\right.
$$

Proof. It is known that, for any nonempty subset $K \subset \llbracket 1, d \rrbracket$, we have the inequality $\|\cdot\|_{K, \star} \leq$ $\|\cdot\|_{\star, K}[11$, Proposition 2.2]. From the definition (10) of the generalized top- $k$ dual norm, and the definition (8) of the dual coordinate- $k$ norm, we obtain (30b). By taking the dual norms, we get (30a).

The norms for which the equality $\|\cdot\|\left\|_{K, \star}=\right\|\|\cdot\|_{\star, K}$ holds true for all nonempty subsets $K \subset$ $\llbracket 1, d \rrbracket$, are the orthant-monotonic norms ([7, Characterization 2.26], [11, Theorem 3.2]). Therefore, if the norm $\||\| \cdot|$ is orthant-monotonic, from the definition (10) of the generalized top- $k$ dual norm, 
we get that the inequality (30b) becomes an equality. Then, the inequality (30a) also becomes an equality by taking the dual norm as in (26). Thus, we have obtained (31).

This ends the proof.

Proposition 14 If the dual norm $\|\cdot\|_{\star}$ is orthant-strictly monotonic, we have that

$$
\ell_{0}(y)=l \Longrightarrow\left\{\begin{array}{l}
\|y\|_{(1), \star}^{\mathcal{R}}<\cdots<\|y\|_{(l-1), \star}^{\mathcal{R}}<\|y\|_{(l), \star}^{\mathcal{R}}=\cdots=\|y\|_{(d), \star}^{\mathcal{R}}=\|y\|_{\star} \\
\|y\|_{\star,(1)}^{\mathrm{tn}}<\cdots<\|y\|_{\star,(l-1)}^{\mathrm{tn}}<\|y\|_{\star,(l)}^{\mathrm{tn}}=\cdots=\|y\|_{\star,(d)}^{\mathrm{tn}}=\|y\|_{\star} .
\end{array}\right.
$$

Proof. We consider $y \in \mathbb{R}^{d}$. We put $L=\operatorname{supp}(y)$ and we suppose that $\ell_{0}(y)=|L|=l$.

Since the norm $\|\cdot\|_{\star}$ is orthant-strictly monotonic, it is orthant-monotonic and so is $\|\cdot\| \|$ by Proposition 11. By (31) in Proposition 13, we get that $\|\cdot\|\left\|_{(j)}^{\mathcal{R}}=\right\| \cdot\|\cdot\|_{\star,(j)}^{\star s n}$ and $\|\cdot\| \cdot\left\|_{(j), \star}^{\mathcal{R}}=\right\| \cdot \|_{\star,(j)}^{\text {tn }}$, for $j \in \llbracket 0, d \rrbracket$ (with the convention that these are the null seminorms in the case $j=0$ ). Therefore, we can translate all the results, obtained in [4], with coordinate- $k$ and dual coordinate- $k$ norms, into results regarding generalized top- $k$ and $k$-support dual norms. As an application, by [4, Equation (18)], we get, from $\ell_{0}(y)=l$, that

$$
\|y\|_{\star,(1)}^{\text {tn }} \leq \cdots \leq\|y\|_{\star,(j)}^{\text {tn }} \leq\|y\|_{\star,(j+1)}^{\text {tn }} \leq \cdots \leq\|y\|_{\star,(d)}^{\text {tn }}=\|y\|_{\star}, \forall y \in \mathbb{R}^{d} .
$$

We now prove (32) in two points.

We first show that $\|y\|_{\star,(l)}^{\mathrm{tn}}=\cdots=\|y\|_{\star,(d)}^{\mathrm{tn}}=\|y\|_{\star}$ (the right hand side of (32)). Since $y=y_{L}$, by definition of the set $L=\operatorname{supp}(y)$, we have that $\|y\|_{\star}=\|\| y_{L}\left\|_{\star} \leq \sup _{|K| \leq l}\right\| y_{K}\|\|_{\star}=\|y\|_{\star,(l)}^{\operatorname{tn}}$ by the very definition (10) of the generalized top- $l$ dual norm $\|\cdot\|_{\star,(l)}^{\text {tn }}$. By (33), we conclude that $\|y\|_{\star,(l)}^{\mathrm{tn}}=\cdots=\|y\|_{\star,(d)}^{\mathrm{tn}}=\|y\|_{\star}$.

Second, we show that $\|y\|_{\star,(1)}^{\mathrm{tn}}<\cdots<\|y\|_{\star,(l-1)}^{\mathrm{tn}}<\|y\|_{\star,(l)}^{\mathrm{tn}}$ (the left hand side of $\left.(32)\right)$. There is nothing to show for $l=0$. Now, for $l \geq 1$ and for any $k \in \llbracket 0, l-1 \rrbracket$, we have

$$
\begin{array}{rlrl}
\|y\|_{\star,(k)}^{\text {tn }} & =\sup _{|K| \leq k}\left\|y_{K}\right\| \|_{\star} & \text { (by definition (10) of the generalized top- } k \text { dual norm) } \\
& =\sup _{|K| \leq k}\left\|y_{K \cap L}\right\|_{\star} & \text { (because } \left.y_{L}=y \text { by definition of the set } L=\operatorname{supp}(y)\right) \\
& =\sup _{\left|K^{\prime}\right| \leq k, K^{\prime} \subset L}\left\|y_{K^{\prime}}\right\|_{\star} & \text { (by setting } \left.K^{\prime}=K \cap L\right) \\
& =\sup _{|K| \leq k, K \subset L}\left\|y_{K}\right\|_{\star} & & \text { (the same but with } K \text { instead of } K^{\prime} \text { ) } \\
& =\sup _{|K| \leq k, K \subsetneq L}\left\|y_{K}\right\|_{\star} & \text { (because }|K| \leq k \leq l-1<l=|L| \text { implies that } K \neq L \text { ) } \\
& <\sup _{|K| \leq k, j \in L \backslash K}\left\|y_{K \cup\{j\}}\right\|_{\star}
\end{array}
$$


because the set $L \backslash K$ is nonempty (having cardinality $|L|-|K|=l-|K| \geq k+1-|K| \geq 1$ ), and because, since the norm $\|\cdot\|_{\star}$ is orthant-strictly monotonic, using Item ( $\boldsymbol{a}$ ) in Proposition 12, we obtain that $\left\|y_{K}\right\|_{\star}<\left\|y_{K \cup\{j\}}\right\|_{\star}$ as $y_{K} \neq y_{K \cup\{j\}}$ for at least one $j \in L \backslash K$ since $L=\operatorname{supp}(y)$

$$
\begin{aligned}
& \leq \sup _{|J| \leq k+1, J \subset L}\left\|y_{J}\right\|_{\star} \\
& \quad\left(\text { as all the subsets } K^{\prime}=K \cup\{j\} \text { are such that } K^{\prime} \subset L \text { and }\left|K^{\prime}\right|=k+1\right) \\
& \leq\|y\|_{\star,(k+1)}^{\text {tn }}
\end{aligned}
$$

by definition (10) of the generalized top- $(k+1)$ dual norm (in fact the last inequality is easily shown to be an equality as $\left.y_{L}=y\right)$. Thus, for any $k \in \llbracket 0, l-1 \rrbracket$, we have established that $\|y\|_{\star,(k)}^{\mathrm{tn}}<\|y\|_{\star,(k+1)}^{\mathrm{tn}}$.

This ends the proof.

\section{B Background on the Fenchel conjugacy on $\mathbb{R}^{d}$}

We review concepts and notations related to the Fenchel conjugacy (we refer the reader to [18]). For any function $h: \mathbb{R}^{d} \rightarrow \overline{\mathbb{R}}$, its epigraph is epi $h=\left\{(w, t) \in \mathbb{R}^{d} \times \mathbb{R} \mid h(w) \leq t\right\}$, its effective domain is dom $h=\left\{w \in \mathbb{R}^{d} \mid h(w)<+\infty\right\}$. A function $h: \mathbb{R}^{d} \rightarrow \overline{\mathbb{R}}$ is said to be convex if its epigraph is a convex set, proper if it never takes the value $-\infty$ and that $\operatorname{dom} h \neq \emptyset$, lower semi continuous (lsc) if its epigraph is closed, closed if it either lsc and nowhere having the value $-\infty$, or is the constant function $-\infty[18, \mathrm{p} .15]$. Closed convex functions are the two constant functions $-\infty$ and $+\infty$ united with all proper convex lsc functions. $^{10}$

For any functions $f: \mathbb{R}^{d} \rightarrow \overline{\mathbb{R}}$ and $g: \mathbb{R}^{d} \rightarrow \overline{\mathbb{R}}$, we denote ${ }^{11}$

$$
\begin{gathered}
f^{\star}(y)=\sup _{x \in \mathbb{R}^{d}}(\langle x, y\rangle+(-f(x))), \quad \forall y \in \mathbb{R}^{d}, \\
g^{\star^{\prime}}(x)=\sup _{y \in \mathbb{Y}}(\langle x, y\rangle+(-g(y))), \quad \forall x \in \mathbb{R}^{d}, \\
f^{\star \star^{\prime}}(x)=\sup _{y \in \mathbb{R}^{d}}\left(\langle x, y\rangle+\left(-f^{\star}(y)\right)\right), \quad \forall x \in \mathbb{R}^{d} .
\end{gathered}
$$

It is proved that the Fenchel conjugacy (indifferently $f \mapsto f^{\star}$ or $g \mapsto g^{\star^{\prime}}$ ) induces a one-to-one correspondence between the closed convex functions on $\mathbb{R}^{d}$ and themselves [18, Theorem 5].

In [20, p. 214-215], the notions ${ }^{12}$ of (Moreau) subgradient and of (Rockafellar) subdifferential are defined for a convex function. Following the definition of the subdifferential of a

\footnotetext{
${ }^{10}$ In particular, any closed convex function that takes at least one finite value is necessarily proper convex lsc.

${ }^{11}$ In convex analysis, one does not use the notation $\star^{\prime}$ in $(34 \mathrm{~b})$ and ${ }^{\star}{ }^{\prime}$ in $(34 \mathrm{c})$, but simply ${ }^{\star}$ and ${ }^{\star \star}$. We use $\star^{\prime}$ and $\star \star^{\prime}$ to be consistent with the notation $(6 \mathrm{~b})$ for general conjugacies.

${ }^{12}$ See the historical note in [19, p. 343].
} 
function with respect to a duality in [1], we define the (Rockafellar-Moreau) subdifferential $\partial f(x)$ of a function $f: \mathbb{R}^{d} \rightarrow \overline{\mathbb{R}}$ at $x \in \mathbb{R}^{d}$ by

$$
\partial f(x)=\left\{y \in \mathbb{R}^{d} \mid f^{\star}(y)=\langle x, y\rangle+(-f(x))\right\} .
$$

When the function $f$ is proper convex and $x \in \operatorname{dom} f$, we recover the classic definition that

$$
\partial f(x)=\left\{y \in \mathbb{R}^{d} \mid\left\langle x^{\prime}-x, y\right\rangle+f(x) \leq f\left(x^{\prime}\right), \forall x^{\prime} \in \operatorname{dom} f\right\} .
$$

\section{References}

[1] M. Akian, S. Gaubert, and V. Kolokoltsov. Invertibility of functional Galois connections. Comptes Rendus Mathematique, 335(11):883-888, 2002.

[2] A. Argyriou, R. Foygel, and N. Srebro. Sparse prediction with the $k$-support norm. In Proceedings of the 25th International Conference on Neural Information Processing Systems - Volume 1, NIPS'12, pages 1457-1465, USA, 2012. Curran Associates Inc.

[3] R. Bhatia. Matrix Analysis. Springer-Verlag, New York, 1997.

[4] J.-P. Chancelier and M. De Lara. Constant along primal rays conjugacies and the $l_{0}$ pseudonorm. Optimization, 0(0):1-32, 2020.

[5] J.-P. Chancelier and M. De Lara. Hidden convexity in the $l_{0}$ pseudonorm. Journal of Convex Analysis, 28(1), 2021.

[6] Z. Fan, H. Jeong, Y. Sun, and M. P. Friedlander. Polar alignment and atomic decomposition. Preprint arXiv:1912.05068, 2019.

[7] D. Gries. Characterization of certain classes of norms. Numerische Mathematik, 10:3041, 1967.

[8] D. Gries and J. Stoer. Some results on fields of values of a matrix. SIAM Journal on Numerical Analysis, 4(2):283-300, 1967.

[9] J.-B. Hiriart-Urruty and H. Le. A variational approach of the rank function. TOP: An Official Journal of the Spanish Society of Statistics and Operations Research, 21(2):207$240,2013$.

[10] J.-B. Hiriart-Urruty and C. Lemaréchal. Convex Analysis and Minimization Algorithms I. Springer-Verlag, Berlin, 1993.

[11] E. Marques de Sà and M.-J. Sodupe. Characterizations of *orthant-monotonic norms. Linear Algebra and its Applications, 193:1-9, 1993. 
[12] J. E. Martínez-Legaz. Generalized convex duality and its economic applications. In S. S. Hadjisavvas N., Komlósi S., editor, Handbook of Generalized Convexity and Generalized Monotonicity. Nonconvex Optimization and Its Applications, volume 76, pages 237-292. Springer-Verlag, 2005.

[13] A. M. McDonald, M. Pontil, and D. Stamos. New perspectives on k-support and cluster norms. Journal of Machine Learning Research, 17(155):1-38, 2016.

[14] L. Mirsky. Symmetric Gauge Functions and Unitarily Invariant Norms. The Quarterly Journal of Mathematics, 11(1):50-59, January 1960.

[15] J. J. Moreau. Inf-convolution, sous-additivité, convexité des fonctions numériques. $J$. Math. Pures Appl. (9), 49:109-154, 1970.

[16] M. Nikolova. Relationship between the optimal solutions of least squares regularized with 10-norm and constrained by k-sparsity. Applied and Computational Harmonic Analysis, 41(1):237 - 265, 2016.

[17] G. Obozinski and F. Bach. A unified perspective on convex structured sparsity: Hierarchical, symmetric, submodular norms and beyond. Preprint, Dec. 2016.

[18] R. T. Rockafellar. Conjugate Duality and Optimization. CBMS-NSF Regional Conference Series in Applied Mathematics. Society for Industrial and Applied Mathematics, 1974.

[19] R. T. Rockafellar and R. J.-B. Wets. Variational Analysis. Springer-Verlag, Berlin, 1998.

[20] T. R. Rockafellar. Convex Analysis. Princeton University Press, Princeton, N.J., 1970.

[21] A. Rubinov. Abstract convexity and global optimization, volume 44 of Nonconvex Optimization and its Applications. Kluwer Academic Publishers, Dordrecht, 2000.

[22] I. Singer. Abstract Convex Analysis. Canadian Mathematical Society Series of Monographs and Advanced Texts. John Wiley \& Sons, Inc., New York, 1997.

[23] K. Tono, A. Takeda, and J.-y. Gotoh. Efficient DC algorithm for constrained sparse optimization. Preprint, January 2017. 